\title{
Full counting statistics of charge transfer in Coulomb blockade systems
}

\author{
D. A. Bagrets and Yu. V. Nazarov \\ Department of Applied Physics and Delft Institute of Microelectronics and Submicrontechnology, Delft University of Technology, \\ Lorentzweg 1, 2628 CJ Delft, The Netherlands
}

(Received 31 July 2002; revised manuscript received 2 December 2002; published 27 February 2003)

\begin{abstract}
Full counting statistics (FCS) of charge transfer in mesoscopic systems has recently become a subject of significant interest, since it proves to reveal an important information about the system which can be hardly assessed by other means. While the previous research mostly addressed the FCS of noninteracting systems, the present paper deals with the FCS in the limit of strong interaction. In this Coulomb blockade limit the electron dynamics is known to be governed by a master equation. We develop a general scheme to evaluate the FCS in such case, this being the main result of the work presented. We illustrate the scheme, by applying it to concrete systems. For generic case of a single resonant level we establish the equivalence of scattering and master equation approach to FCS. Further we study a single Coulomb blockade island with two and three leads attached and compare the FCS in this case with our recent results concerning an open dot either with two and three terminals. We demonstrate that Coulomb interaction suppresses the relative probabilities of large current fluctuations.
\end{abstract}

DOI: 10.1103/PhysRevB.67.085316

PACS number(s): 73.23.Hk, 05.40.-a, 72.70.+m

\section{INTRODUCTION}

The current fluctuations in the various mesoscopic systems have been the subject of both theoretical and experimental research in the last two decades. Traditionally, the attention was focused on the shot noise phenomenon. The shot noise is the main fundamental source of current noise at low temperatures. In classical systems shot noise unambiguously related to the discreteness of the electron charge. In quantum system the shot noise can be used as unique tool to reveal the information about the electron correlations and entanglement of different kind. The investigation of the quantum shot-noise cross-correlations in the multiterminal mesoscopic devices is the new trend in this field, which has attracted much attention as well. The most achievements in the study of the shot-noise phenomena have been summarized in the recent review article. ${ }^{1}$

Alternative way to investigate the current correlations in the mesoscopic systems has proposed in the pioneering work by Levitov et al. ${ }^{2}$ This new fascinating theoretical approach, known as the full counting statistics (FCS), yields not only shot-noise power but also all possible correlations and momenta of charge transfer. The essence of this method is an evaluation of the probability distribution function of the numbers of electrons transferred to the given terminals during the given period of time. The first and the second moments of this distribution correspond to the average currents and the shot-noise correlations, respectively. The probability distribution also contains the fundamental information about large current fluctuations in the system.

Initially, FCS method ${ }^{2}$ made use of the scattering approach to mesoscopic transport. It was assumed that the mesoscopic system was completely characterized by its scattering matrix. This method enabled to study the statistics of the transport through the disordered metallic conductor ${ }^{3}$ and the two-terminal chaotic cavity. ${ }^{4}$ Muzykantskii and Khmelnitskii generalized the original approach to the case of the normal metal/superconducting contacts. The very recent development in this field is the counting statistics of the charge pumping in the open quantum dots. ${ }^{5-7}$

The use of multichannel scattering matrix of the system was crucial to obtain the results of the above mentioned works. However, such approach leads to the difficulties in case of practical layouts, where the scattering matrix is random and cumbersome. They become apparent especially in case of multiterminal geometry. To circumvent these difficulties one evaluates the FCS with the semiclassical Keldysh Green's function method ${ }^{8}$ or with its simplification called the circuit theory of mesoscopic transport. ${ }^{9}$ The Keldysh method to FCS was first proposed by one of the authors in order to treat the effects of the weak localization corrections onto the FCS in the disordered metallic wires. The method proves to be very flexible and has been recently applied to the FCS in superconducting heterostructures, ${ }^{10}$ multiterminal normal metal systems ${ }^{11}$ as well as in the three-terminal superconducting beam splitter. ${ }^{12}$

The above research addressed the FCS of noninteracting electrons. Since the interaction may bring correlations and entanglement of electron states the study of FCS of interacting electrons is both challenging and interesting. In this paper we present an extensive theory of FCS in mesoscopic systems placed in a strong Coulomb blockade limit.

The FCS statistics of electron pumping of interacting electrons has been considered by Andreev and Mishchenko. ${ }^{13}$ However, they treated this problem with very specific assumptions. Namely, they considered quantum dot with almost open contacts, $R \sim R_{\mathrm{Q}}, R_{\mathrm{Q}}$ being a resistance quantum. The Coulomb blockade effect in these circumstances is very week. Our paper concerns conventional limit of highly resistive contacts, $R \gg R_{\mathrm{Q}}$.

Note, that the shot noise in the Coulomb blockade devices has attracted the significant attention. Korotkov ${ }^{14}$ and Hershfield et al. ${ }^{15}$ presented the first theory in the framework of "orthodox" approach to single electron transport. Later on 
Korotkov also studied the frequency dependence of the shot noise by means of Langevin approach both in low- (classical) and very high- (quantum) frequency limits. The frequency dependence of the shot noise in the single electron transistor was also investigated in Refs. 16 and 17. The ferromagnetic single electron transistor was considered by Bulka et. al. ${ }^{18}$ The shot-noise experiments were performed by Birk, Jong, and Schönenberger. ${ }^{19}$ In this work, the nanoparticle in between the scanning tunneling microscope tip and the metallic electrode was used to form the Coulomb blockade island and the quantitative agreement with the theory of Hershfield et al. was found.

The electrons dynamics in Coulomb blockade limit is fortunately relatively simple. When the cotunneling phenomena is disregarded, the evolution of the system is governed by a master equation. The charge transfer is thus a classical stochastic process rather than the quantum mechanical one. Nevertheless the FCS is by no means trivial and has not been studied yet. In the present work we have developed the general approach to FCS in the Coulomb blockade regime. This is the central result of the paper. Our method turns out to be an elegant extension of the usual master equation approach.

We apply the developed scheme to study the FCS in various Coulomb blockade systems. We present the number of different results as well as reestablish some old ones. For a generic model of a single resonance level we establish the equivalence of the new master equation approach to the FCS with the well-known scattering approach by Levitov et al. This equivalence holds under assumption of noninteracting particles, when both descriptions becomes applicable.

Further the scheme is used to study the FCS of charge transfer and shot-noise correlations in the two- and threeterminal Coulomb blockade island. Our consideration is limited to the temperature regime $k_{B} T \gg \Delta E, \Delta E$ being the level spacing in the dot. We compare the results, obtained in Coulomb blockade limit with our previous study of noninteracting electrons in chaotic quantum dots. ${ }^{11}$ Surprisingly, we found, that the FCS has a similar qualitative features both in weakly and strongly interacting regimes. We show that Coulomb interaction suppresses the relative probabilities of big current fluctuations. The previous results for the zerofrequency shot-noise power in the conventional single electron transistor can be evaluated in our approach as the second moment of charge-transfer probability distribution function. Regarding the Coulomb blockade island with three leads attached we show that the auto- and cross-shot-noise correlations exhibit the characteristic Coulomb blockade oscillations as functions of the applied voltages and offset charge.

The paper is organized as follows. In the Sec. II we start by presenting the two physical systems to be treated within the master equation. Based on these prototypes we formulate the general model. We derive our approach to the FCS in the Sec. III. In the Sec. IV we applied the method to study the FCS of the single resonant level and consider its relation to the scattering approach. Two- and three-terminal single electron transistors are considered in Sec. V. We also compare their FCS in the Coulomb blockade limit with that of noninteracting electrons. ${ }^{11}$ We summarize the results in Sec. VI.

\section{SYSTEMS UNDER CONSIDERATION}

The dynamics of various systems can be described by master equation. For our purposes it is convenient to write it down in the matrix form

$$
\frac{\partial}{\partial t}|p, t\rangle=-\hat{L}|p(t)\rangle
$$

where each element $p_{n}(t)$ of the vector $|p(t)\rangle$ corresponds to the probability to find the system in the state $n$. The matrix elements of operator $\hat{L}$ are given by

$$
L_{m n}=\delta_{n m} \gamma_{n}-\Gamma_{m \leftarrow n}, \quad \gamma_{n}=\sum_{m \neq n} \Gamma_{m \leftarrow n} .
$$

Here $\Gamma_{n \leftarrow m}$ stands for the transition rate from the state $m$ to the state $n, \gamma_{n}$ presents the total transition rate from the state $n$. Thus defined operator $\hat{L}$ always has a zero eigenvalue, the corresponding eigenvector being the stationary solution of the master equation.

Coulomb blockade mesoscopic systems always obey Eq. (1). The main advantage of the master equation approach is a possibility of nonpertubative treatment of the interaction effects. In what follows, we first remind the master equation description of two simple systems: single resonant level and many-terminal Coulomb blockade island. On the basis of these examples we will sketch the master equation for the general Coulomb blockade system. This will prepare us to the following section, where we derive the FCS method.

\section{A. Resonant-level model}

An elaborated model of the resonant center was presented in Ref. 20. It was subsequently improved in the work $^{21}$ to include the Coulomb interaction. One of the physical realization is disordered tunneling barrier which is placed between two leads. ${ }^{22}$ At sufficiently low temperatures the main mechanism of transport in this system is the resonant tunneling via localized states formed by impurity centers. Another physical realization is the Coulomb blockade island in the low-temperature regime $k_{B} T \ll \Delta E$, where $\Delta E$ is a mean separation between the energy levels in the dot. ${ }^{23}$ By applying the gate voltage, one can tune the given level to be between the chemical potentials of the leads. (See Fig. 1.) We consider below two limiting cases where one disregards either double occupancy of the level or on-cite Coulomb interaction.

In the strongly interacting case the double occupancy of the resonant level is entirely excluded due to the Coulomb repulsion $U$. Then the system can be found only in two different microscopic states: one with no electrons, and another with a single electron. The transport through the level can be described by master equation approach, provided the applied voltage or the temperature are not too low, i.e., $\max \left\{e V, k_{B} T\right\} \gg \hbar \Gamma_{L(R)}$. Here $\Gamma_{L(R)}$ are the quantummechanical tunneling rates from the left (right) electrode onto the resonant level. We will also assume that at the relevant energy scale, given by $\max \left\{e V, k_{B} T\right\}$, the rates $\Gamma_{L(R)}$ are energy independent. 


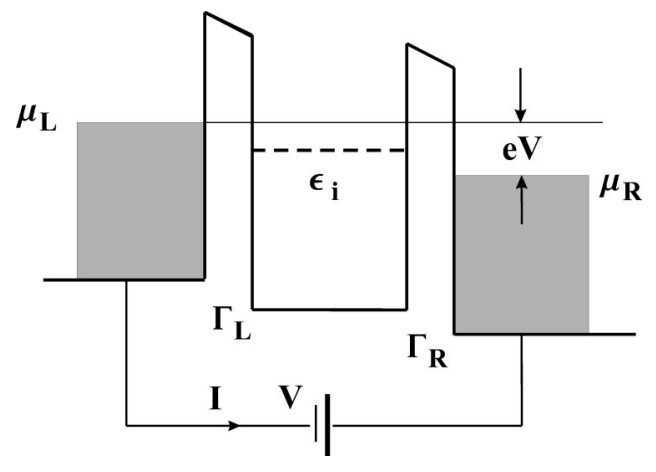

FIG. 1. The single resonant-level system, formed by the two tunnel barriers. The resonant level in the quantum well is shown by the dashed line.

Under above assumptions the transition rates in Eq. (1) are given by

$$
\begin{gathered}
\Gamma_{1 \leftarrow 0}=2 \Gamma_{L} f_{L}\left(\epsilon_{i}\right)+2 \Gamma_{R} f_{R}\left(\epsilon_{i}\right), \\
\Gamma_{0 \leftarrow 1}=\Gamma_{L}\left[1-f_{L}\left(\epsilon_{i}\right)\right]+\Gamma_{R}\left[1-f_{R}\left(\epsilon_{i}\right)\right] .
\end{gathered}
$$

The microscopic states $\{0\}$ and $\{1\}$ denote the situation with no and one electron, respectively. Fermi function $f_{L(R)}(\epsilon)$ $=\left\{1+\exp \left[\left(\epsilon-\mu_{L(R)}\right) / k T\right]\right\}^{-1}$ accounts for the filling in the left (right) lead and $\epsilon_{i}$ is the position of the resonant level. The factor 2 in the rate $\Gamma_{1 \leftarrow 0}$ stems from the fact that two quantum states, with spin up and down, are available for tunneling. The description in terms of rates (3) is correct when the Coulomb repulsion is strong enough, $U$ $\gg \max \left\{e V, k_{B} T\right\}$.

The opposite limit is the case of vanishing Coulomb interaction. In this case the spin-up and spin-down channels can be treated independently. Each of them can be described by the master equation, provided the same condition as before is fulfilled: $\max \left\{e V, k_{B} T\right\} \gg \hbar \Gamma_{L(R)}$. For both spin directions the rates are written as

$$
\begin{gathered}
\Gamma_{1 \leftarrow 0}=\Gamma_{L} f_{L}\left(\epsilon_{i}\right)+\Gamma_{R} f_{R}\left(\epsilon_{i}\right), \\
\Gamma_{0 \leftarrow 1}=\Gamma_{L}\left[1-f_{L}\left(\epsilon_{i}\right)\right]+\Gamma_{R}\left[1-f_{R}\left(\epsilon_{i}\right)\right] .
\end{gathered}
$$

Here the indices $\{0\}$ and $\{1\}$ denote the filling factor of the level by electon with a chosen spin.

Disregarding of Coulomb interaction is not adequate for a realistic system. However, the latter model is worth to consider as well. The point is that the statistics of the charge transfer in this case can be also evaluated in the framework of the noninteracting scattering approach, ${ }^{2}$ thus providing the way to establish the consistency of two approaches to FCS.

\section{B. Many-terminal Coulomb blockade island}

The electrical circuit incorporating the Coulomb blockade island with several terminals is shown in Fig. 2. This circuit is an extension of the usual single electron transistor. ${ }^{24}$ At the present stage of nanotechnology the mesoscopic system, associated with this circuit, can be realized with the use of two-dimensional (2D) electron gas in the GaAs/AlGaAs heterostructures.

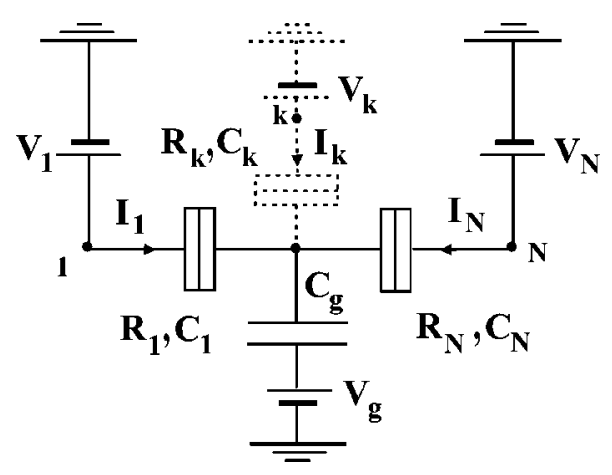

FIG. 2. The equivalent circuit of the $N$-terminal Coulomb blockade island. Each junction $k$ is biased by the external voltage source $V_{k}$. The island is also capacitatively coupled with the gate voltage $V_{g}$.

The essential elements of the circuit shown in Fig. 2 are the resistances $R_{k}$ of the contacts, the mutual capacitances $C_{k}$ between the leads and the island and the external dc voltage sources $V_{k}$. Correspondingly, $C_{g}$ and $V_{g}$ denote the gate capacitance and gate voltage, which is used to vary the offset change on the island. We assume that the island is placed in the Coulomb blockade regime, $R_{k} \gg R_{Q}$ $=2 \pi \hbar / e^{2}$. In order the Coulomb blockade effect will be observable the condition $k_{B} T \ll E_{c}=e^{2} / 2 C_{\Sigma}$ is also required. Here $E_{c}$ is a charging energy of the island, $C_{\Sigma}=\sum_{i=1}^{N} C_{k}$ $+C_{g}$ is a sum capacitance of the system and $N \geqslant 2$ is a number of leads attached to the dot. We also assume the temperature to be rather high, $k_{B} T \gg \Delta E$, with $\Delta E$ being the mean level spacing in the dot, so that the discreteness of the energy spectrum in the island is not important. The possible effects of cotunneling will not be discussed in the paper. Therefore, the characteristic scale of applied voltage $e V$ is assumed to be greater than the Coulomb blockade threshold, $e V \geqslant E_{c}$.

Under the above conditions the multiterminal Coulomb blockade island is fairly well described by the "orthodox" Coulomb blockade theory. One can consider the excess number of electrons on the island $(-n)$ as a good quantum number, corresponding to the macroscopic state of the system. The tunneling of electrons will occur one by one, increasing or decreasing the charge $Q_{0}=n e$ on the island by $\pm e$. The corresponding tunneling rate $\Gamma_{n \pm 1 \leftarrow n}^{(k)}$ across the junction $k$ is expressed via the electrostatic energy difference $\Delta E_{n \pm 1 \leftarrow n}^{(k)}$ between the initial $(n)$ and final $(n \pm 1)$ configurations

$$
\Gamma_{n \pm 1 \leftarrow n}^{(k)}=\frac{1}{e^{2} R_{k}} \frac{\Delta E_{n \pm 1 \leftarrow n}^{(k)}}{1-\exp \left[-\Delta E_{n \pm 1 \leftarrow n}^{(k)} / k_{B} T\right]} .
$$

The evaluation of $\Delta E_{n \pm 1 \leftarrow n}^{(k)}$ can be done along the same lines as in the case of single electron transistor. ${ }^{24}$ The result reads

$$
\Delta E_{n \pm 1 \leftarrow n}^{(k)}= \pm e\left[V_{k}-V_{0}(n)\right]-\frac{e^{2}}{2 C_{\Sigma}},
$$

where $V_{0}(n)$ is the electrostatic potential on the island. It is written as 


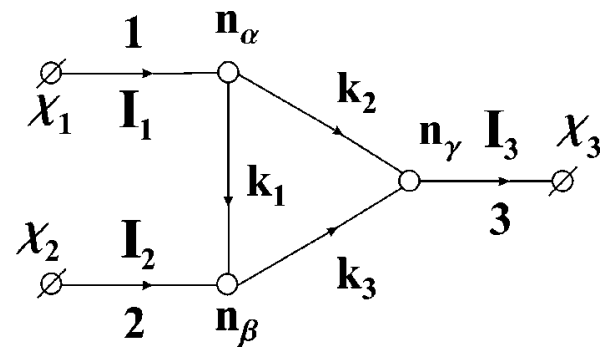

FIG. 3. The graph of general model (see the main text). The terminals are connected with the system via external junctions 1,2 , and 3. The nodes $\alpha, \beta$, and $\gamma$ are either resonant levels or dots, linked with each other by internal junctions $k$ 's. The arrows denote the conventional direction of a current through each junction.

$$
V_{0}(n)=\frac{1}{C_{\Sigma}}\left(e n+C_{g} V_{g}\right)+\frac{1}{C_{\Sigma}} \sum_{i=1}^{N} \widetilde{C}_{i} V_{i}
$$

Here $\widetilde{C}_{i} \equiv C_{i}+C_{g} / N$ and we also assumed that the dot is biased in such a way, that external voltages are subjected to the condition $\sum_{i=1}^{N} V_{i}=0$. In this case the gate voltage $V_{g}$ can be used to influence the offset charge $q=C_{g} V_{g}$ on the island in a controlled way.

Neglecting the quantum correlations between different tunneling processes, we may write down the master equation (1). It connects the states with different island charge, the total transition rate from the state $n$ to $n \pm 1$ being the sum of tunneling rates over all junctions: $\Gamma_{n \pm 1 \leftarrow n}=\sum_{k=1}^{N} \Gamma_{n \pm 1 \leftarrow n}^{(k)}$.

\section{General model}

We now outline the general model which is an extension of the preceding two. The possible physical realization of this model includes an array of Coulomb blockade quantum dots and a mesoscopic system with a number of resonant levels. We assume that all the relevant conditions, mentioned previously, are satisfied and therefore the description in terms of master equation is valid. It is convenient schematically to represent the system as a graph (see Fig. 3), with each node $\alpha$ corresponding either to the single dot, the single resonant level or the external terminal. The line $k=(\alpha, \beta)$, connecting the nodes $\alpha$ and $\beta$ is associated with the possible electron transfer. Let $M$ be the total number of nodes in this graph and $L$ is a total number of lines. For a many-dot system each line $k$ corresponds to the tunnel junction. For systems with many resonant levels it corresponds to the possible transition between different levels, so that it does not necessary correspond to electron transfer in space. The lines are assumed to be directed, thus specifying the sign convention for a current $I_{k}$ through the line $k$. There are $N$ external junction $k=1, \ldots, N,(N \leqslant L)$, connecting the terminals with the system. The currents through these junctions are directly measurable and hence are of our interest.

The macroscopic or microscopic state of the general model is given by a set of occupation numbers $|n\rangle$ $=\left|n_{1}, \ldots, n_{M}\right\rangle ; n_{\alpha}$ is equal to any integer for the array of quantum dots and refers to the excess charge on the island $\alpha$; in case of many resonant-levels system $n_{\alpha}$ denotes the occupation number of the given level.

We now consider the general properties of the master equation (1) describing the above model. Owing to the fact $\Sigma_{n} L_{n m}=0$, the $\hat{L}$ operator has the right, $\left|p_{0}\right\rangle$, and the left, $\left\langle q_{0}\right|$, eigenvectors corresponding to zero eigenvalue

$$
\hat{L}\left|p_{0}\right\rangle=0,\left\langle q_{0}\right| \hat{L}=0 .
$$

We assume that they are unique. This means that the system does not get stuck in any metastable state. The vector $\left|p_{0}\right\rangle$ gives the steady probability distribution and $\left\langle q_{0}\right|$ $=(1,1, \ldots, 1)$. Since we are interested, in general, only in the permanent, but not the transient processes in the system it is naturally to restrict the consideration only to absorbing states $n$. Thus we will exclude all the transient ones $n^{\prime}$, for which $\Gamma_{n n^{\prime}}>0$ but at the same time $\Gamma_{n^{\prime} n}=0$. We assume that the $\hat{L}$ operator, bounded to the absorbing states, has a complete set of left and write eigenvectors

$$
\hat{L}\left|p_{k}\right\rangle=\left|p_{k}\right\rangle \lambda_{k}, \quad \lambda_{k}\left\langle q_{k}\right|=\left\langle q_{k}\left|\hat{L}, \quad \sum_{k}\right| p_{k}\right\rangle\left\langle q_{k}\right|=\hat{I},
$$

where $\hat{I}$ is a unitary operator in the absorbing subspace. For any physically reasonable $\hat{L}$ operator $\lambda_{0}=0$ and $\operatorname{Re} \lambda_{k}>0$ for $k \neq 0$.

For the following it is also useful to represent $\hat{L}$ operator in the form

$$
\hat{L}=\hat{\gamma}-\hat{\Gamma}, \quad \hat{\Gamma}=\sum_{k=1}^{L}\left(\hat{\Gamma}_{k}^{(+)}+\hat{\Gamma}_{k}^{(-)}\right),
$$

where $\hat{\gamma}$ is the diagonal operator in the basis $|n\rangle$ of the system configuration and $\hat{\Gamma}_{k}^{( \pm)}$are associated with the tunneling transitions through the "junction" $k=(\alpha, \beta)$ :

$$
\hat{\gamma}=\sum_{\{n\}}|n\rangle \gamma(n)\left\langle n\left|, \quad \hat{\Gamma}_{k}^{( \pm)}=\sum_{\{n\}}\right| n^{\prime}\right\rangle \Gamma_{k}^{( \pm)}(n)\langle n| .
$$

The state $\left|n^{\prime}\right\rangle=\left|n_{1}, \ldots, n_{\alpha}^{\prime}, \ldots, n_{\beta}^{\prime}, \ldots, n_{M}\right\rangle$ results from the state $|n\rangle$ by appropriate changing the corresponding occupation numbers: $n_{\alpha}^{\prime}=n_{\alpha}-\sigma_{k}, n_{\beta}^{\prime}=n_{\beta}+\sigma_{k}$, where $\sigma_{k}$ $= \pm 1$ denotes the direction of the transition.

\section{THE FCS IN THE MASTER EQUATION}

In this section we derive the central result for the FCS of the charge transfer in the system, which dynamics obeys the master equation. We will solve this problem by making use of the property of the system, that its random evolution in time is the Markov stochastic process.

In what follows we will partially use notations of the book. ${ }^{25}$ Let us consider the time interval $[-T / 2, T / 2]$. Suppose the system undergoes $s$ transitions at random time moments $\tau_{i}$, so that

$$
+T / 2>\tau_{1}>\tau_{2}>\cdots>\tau_{s-1}>\tau_{s}>-T / 2 .
$$


This gives an elementary random sample $\zeta_{s}$ $=\left(\tau_{1}, k_{1}, \sigma_{1}, \ldots, \tau_{s}, k_{s}, \sigma_{s}\right)$. It corresponds to the set of subsequent events, when at time $\tau_{i}$ the tunneling happens via the junction $k_{i}, \sigma_{i}= \pm 1$ being the direction of the transition. The samples $\zeta_{s}$ constitute the set $\Omega$ of all possible random samples.

Then one defines the measure (or the probability) $d \mu(\zeta)$ at the set $\Omega$. For this purpose we may very generally introduce the sequence of non-negative probabilities $Q_{s}\left(\left\{\tau_{i}, k_{i}, \sigma_{i}\right\}\right) \equiv Q\left(\tau_{1}, k_{1}, \sigma_{1}, \ldots, \tau_{s}, k_{s}, \sigma_{s}\right) \geqslant 0$ defined in $\Omega$ so that

$$
d \mu(\zeta)=Q_{0}+\sum_{s=1}^{+\infty} \sum_{\left\{k_{i}, \sigma_{i}\right\}} Q_{s}\left(\left\{\tau_{i}, k_{i}, \sigma_{i}\right\}\right) d \tau_{1} \cdots d \tau_{s} .
$$

The functions $Q$ are normalized according to the condition

$$
\begin{aligned}
\int_{\Omega} d \mu(\zeta) \equiv & Q_{0}+\sum_{s=1}^{+\infty} \sum_{\left\{k_{i}, \sigma_{i}\right\}} \int \cdots \int_{T / 2>\tau_{1}>\ldots>\tau_{s}>-T / 2} \\
& \times Q_{s}\left(\left\{\tau_{i}, k_{i}, \sigma_{i}\right\}\right) \prod_{i=1}^{s} d \tau_{i}=1
\end{aligned}
$$

Each term in Eq. (13) corresponds to the probability of an elementary sample $\zeta_{s}$.

To accomplish the preliminaries, we remind the concept of a stochastic process. Mathematically speaking, it can be any integrable function $\check{A}(t) \equiv A(t, \zeta)$ defined at the set $\Omega$ and parametrically depending on time. It is sometimes convenient to omit the explicit $\zeta$ dependence. We will use a "check" in this case to stress that the quantity in question is a random variable. Each stochastic process $A(t, \zeta)$ generates the sequence of time-dependent functions $\left\{A_{0}(t), A_{1}\left(t, \tau_{1}, k_{1}, \sigma_{1}\right), \ldots, A_{s}\left(t,\left\{\tau_{i}, k_{i}, \sigma_{i}\right\}\right)\right\}$. Its average $\langle\check{A}(t)\rangle_{\Omega}$ over the space $\Omega$ is defined as

$$
\begin{aligned}
\langle\check{A}(t)\rangle_{\Omega}= & \int_{\Omega} A(t, \zeta) d \mu(\zeta) \equiv A_{0}(t) Q_{0} \\
& +\sum_{s=1}^{+\infty} \sum_{\left\{k_{i}, \sigma_{i}\right\}} \int \cdots \int_{T / 2>\tau_{1}>\ldots>\tau_{s}>-T / 2} \\
& \times A_{s}\left(t,\left\{\tau_{i}, k_{i}, \sigma_{i}\right\}\right) Q_{s}\left(\left\{\tau_{i}, k_{i}, \sigma_{i}\right\}\right) \prod_{i=1}^{s} d \tau_{i}
\end{aligned}
$$

The analogous expression should be used, for instance, to define the correlations $\left\langle\check{A}\left(t_{1}\right) \check{B}\left(t_{2}\right)\right\rangle_{\Omega}$ between any two stochastic processes.

For the subsequent analysis we define the random process $\check{I}^{(k)}(t)$, corresponding to the classical current through the external junction $k \leqslant N$ :

$$
I^{(k)}\left(t, \zeta_{s}\right)=\sum_{i=1}^{s} e \sigma_{i} \delta\left(t-\tau_{i}\right) \delta\left(k-k_{i}\right)
$$

Here $\sigma_{m}$ is included to take into account the direction of the jump and $\delta\left(k-k_{i}\right) \equiv \delta_{k, k_{i}}$ is the Kronecker $\delta$ symbol. Given this definition at hand, we introduce the generating functional $S\left[\left\{\chi_{i}(t)\right\}\right]$ depending on $N$ counting fields $\chi_{i}(\tau)$, each of them associated with a given terminal $i$ :

$\exp \left(-S\left[\left\{\chi_{i}(t)\right\}\right]\right)=\left\langle\exp \left\{i \sum_{n=1}^{N} \int_{-\infty}^{+\infty} d \tau \chi_{n}(\tau) \check{I}^{(n)}(\tau) / e\right\}\right\rangle_{\Omega}$

with the average defined by Eq. (15). We will refer to $S\left[\left\{\chi_{i}(t)\right\}\right]$ as the action. Its evaluation is the main goal of this section. The $m$-order functional derivatives of $S\left[\left\{\chi_{i}(t)\right\}\right]$ with respect to $\chi_{i}$ give the irreducible $m$-order current correlations. First derivatives correspond to the average currents through terminals; the second derivatives give the shot noise and noise correlations. In the low-frequency limit of current correlations one may use the time-independent counting fields $\chi_{i}$. In this case the action $S\left[\left\{\chi_{i}\right\}\right]$ allows to express the probability of $N_{i}$ electrons to be transferred through the terminal $i$ during the time interval $T$

$$
P\left(\left\{N_{i}\right\}\right)=\int_{-\pi i=1}^{\pi} \prod_{i}^{N} \frac{d \chi_{i}}{2 \pi} e^{-S\left(\left\{\chi_{i}\right\}\right)-i \Sigma_{i} N_{i} \chi_{i}}
$$

The above definitions were rather general than constructive, since the probabilities $Q$ have not been specified so far. To proceed, one has to relate them to transition rates of the master equation. We assume that at initial time $t=-T / 2$ the system was in the state $\left\{n^{(s)}\right\}$. Then random sample $\zeta_{s}$ determines the evolution of charge configuration $\left\{n^{(s)}\right\}$ $\rightarrow\left\{n^{(s-1)}\right\} \cdots\left\{n^{(1)}\right\} \rightarrow\left\{n^{(0)}\right\}$ for subsequent moments of time. The choice of $\zeta_{s}$ specifies that the transition between neighboring charge states $\left\{n^{(i)}\right\}$ and $\left\{n^{(i-1)}\right\}$ occurs at time $\tau_{i}$ via the junction $k_{i}=\left(\alpha_{i}, \beta_{i}\right)$. Therefore, the sequence $\left\{n^{(i)}\right\}$ is given by the relation $n_{\alpha_{i}}^{(i-1)}=n_{\alpha_{i}}^{(i)}-\sigma_{k_{i}}, n_{\beta_{i}}^{(i-1)}$ $=n_{\beta_{i}}^{(i)}+\sigma_{k_{i}}$, and $n_{\gamma}^{(i-1)}=n_{\gamma}^{(i)}$ for all $\gamma \neq \alpha_{i}$ and $\beta_{i}$. To determine the probability $Q_{s}\left(\left\{\tau_{i}, k_{i}, \sigma_{i}\right\}\right)$ we note that (i) the sample $\zeta_{s}$ constitutes the Markov chain (ii) the conditional probability of the system to remain at state $n^{(i)}$ between the times $\tau_{i+1}$ and $\tau_{i}$ is proportional to $\exp \left[-\gamma\left(n^{(i)}\right)\left(\tau_{i}-\tau_{i+1}\right)\right]$; (iii) the probability that the transition occurs via the junction $k_{i}$ during the time interval $d \tau_{i}$ at the moment $\tau_{i}$ is given by $\Gamma_{k_{i}}^{\left(\sigma_{i}\right)}\left(n^{(i)}\right) d \tau_{i}$. These arguments suggest that $Q$ 's have the form

$$
\begin{aligned}
Q_{0} & =Z_{0}^{-1} \exp \left[-\gamma\left(n^{(s)}\right) T\right] \\
Q_{s}\left(\left\{\tau_{i}, k_{i}, \sigma_{i}\right\}\right)= & Z_{0}^{-1} \exp \left[-\gamma\left(n^{(0)}\right)\left(T / 2-\tau_{1}\right)\right] \\
& \times \Gamma_{k_{1}}^{\left(\sigma_{1}\right)}\left(n^{(1)}\right) \exp \left[-\gamma\left(n^{(1)}\right)\left(\tau_{1}-\tau_{2}\right)\right] \\
& \times \Gamma_{k_{2}}^{\left(\sigma_{2}\right)}\left(n^{(2)}\right) \cdots \exp \left[-\gamma\left(n^{(s-1)}\right)\right. \\
& \left.\times\left(\tau_{s-1}-\tau_{s}\right)\right] \Gamma_{k_{s}}^{\left(\sigma_{s}\right)}\left(n^{(s)}\right) \exp \left[-\gamma\left(n^{(s)}\right)\right. \\
& \left.\times\left(\tau_{s}+T / 2\right)\right]
\end{aligned}
$$


where the constant $Z_{0}$ should be found from the normalization condition Eq. (14). As we will see below, $Z_{0}=1$.

The above correspondence between the random Markov chain $\zeta_{s}$ and the probabilities $Q$ 's, Eq. (19), allows one to evaluate the generating function Eq. (17). By definition Eq. (16) for any given $\zeta_{s}$ we have

$$
\begin{gathered}
\exp \left\{i \sum_{n=1}^{N} \int_{-\infty}^{+\infty} d \tau \chi_{n}(\tau) I^{(n)}\left(\tau, \zeta_{s}\right) / e\right\} \\
=\prod_{i=1}^{s} \exp \left\{i \sigma_{i} \chi_{k_{i}}\left(\tau_{i}\right)\right\} .
\end{gathered}
$$

It is assumed here that $\chi_{k_{i}}=0$ if the transition occurs via internal junction, $k_{i}>N$, thus no physically measurable current is generated in this case. The averaging of the latter expression over all possible configurations $\Omega$ with the weight $d \mu(\zeta)$ yields

$$
\begin{aligned}
Z\left[\left\{\chi_{i}(\tau)\right\}\right] \equiv & \exp \left(-S\left[\left\{\chi_{i}(\tau)\right\}\right]\right)=Q_{0} \\
& +\sum_{s=1}^{+\infty} \sum_{\left\{k_{i}, \sigma_{i}\right\}} \int \cdots \int_{T / 2>\tau_{1}>\ldots>\tau_{s}>-T / 2} \\
& \times Q_{s}^{\chi}\left(\left\{\tau_{i}, k_{i}, \sigma_{i}\right\}\right) \prod_{i=1}^{s} d \tau_{i} .
\end{aligned}
$$

The resulting expression resembles the normalization condition (14). Here the $\chi$-dependent functions $Q_{s}^{\chi}\left(\left\{\tau_{i}, k_{i}, \sigma_{i}\right\}\right)$ are defined similar to probabilities (19) with the only crucial difference that the rates $\Gamma_{k}^{(\sigma)}(n)$ should be replaced by $\Gamma_{k}^{(\sigma)}(n) \exp \left\{i \sigma_{k} \chi_{k}\left(\tau_{k}\right)\right\}$ if $k \leqslant N$.

Expression (20) can be written in the more compact and elegant way. For that, we introduce the $\chi$-dependent linear operator $\hat{L}_{\chi}$ defined as

$$
\begin{gathered}
\hat{L}_{\chi}(\tau)=\hat{\gamma}-\hat{\Gamma}_{\chi}(\tau), \\
\hat{\Gamma}_{\chi}(\tau)=\sum_{k=1}^{N}\left(\hat{\Gamma}_{k}^{(+)} e^{i \chi_{k}(\tau)}+\hat{\Gamma}_{k}^{(-)} e^{-i \chi_{k}(\tau)}\right) \\
+\sum_{k=N+1}^{L}\left(\hat{\Gamma}_{k}^{(+)}+\hat{\Gamma}_{k}^{(-)}\right) .
\end{gathered}
$$

In line with consideration above we multiplied each operator $\hat{\Gamma}_{k}^{( \pm)}(k=1, \ldots, N)$, that corresponds to the transition through the external junction, by an extra $\chi$-dependent factor $e^{i \chi_{k}(\tau)}$. The diagonal part and internal transition operators $\hat{\Gamma}_{k}^{( \pm)}$with $k>N$ remained unchanged. Then we consider the evolution operator $\hat{U}_{\chi}\left(t_{1}, t_{2}\right)$ associated with Eq. (21). Since $\hat{L}_{\chi}(\tau)$ is in general time-dependent, $\hat{U}_{\chi}\left(t_{1}, t_{2}\right)$ is given by the time-ordered exponent

$$
\hat{U}_{\chi}\left(t_{1}, t_{2}\right)=T_{\tau} \exp \left\{-\int_{t_{2}}^{t_{1}}\left[\hat{\gamma}(\tau)-\hat{\Gamma}_{\chi}(\tau)\right] d \tau\right\} .
$$

The similar construction is widely used in quantum statistics. The difference in the present case is that the operator
$\hat{U}_{\chi}\left(t_{1}, t_{2}\right)$ at $\chi=0$ gives the evolution of probability rather than the amplitude of probability.

With the use of evolution operator (22) the generating function Eq. (20) can be cast into the form

$$
Z\left[\left\{\chi_{i}(\tau)\right\}\right]=\left\langle q_{0}\left|\hat{U}_{\chi}(T / 2,-T / 2)\right| n_{s}\right\rangle .
$$

To prove it we argue as follows. We exploit the fact that $\hat{\gamma}(\tau)$ and $\hat{\Gamma}(\tau)$ commute under the sign of time ordering in Eq. (22) and regard $\hat{\Gamma}(\tau)$ as a perturbation. This gives the matrix element $\left\langle q_{0}\left|\hat{U}_{\chi}(T / 2,-T / 2)\right| n_{s}\right\rangle$ in the form of series

$$
\begin{aligned}
\left\langle q_{0}\left|\hat{U}_{\chi}(T / 2,-T / 2)\right| n_{s}\right\rangle & \\
= & \left\langle q_{0}\left|e^{-\hat{\gamma} T}\right| p_{0}\right\rangle+\sum_{s=1}^{+\infty}\left\langle q_{0}\right| T_{\tau} \exp \left\{-\int_{-T / 2}^{T / 2} \hat{\gamma}(\tau) d \tau\right\} \\
& \times \sum_{\mathbf{k}_{s} \boldsymbol{\sigma}_{s}} \int \cdots \int_{T / 2>\tau_{1}>\ldots>\tau_{s}>-T / 2} \hat{\Gamma}_{k_{1}}^{\left(\sigma_{1}\right)}\left(\tau_{1}\right) \\
& \times e^{i \sigma_{1} \chi_{k_{1}}\left(\tau_{k_{1}}\right)} \cdots \hat{\Gamma}_{k_{s}}^{\left(\sigma_{s}\right)}\left(\tau_{s}\right) e^{i \sigma_{s} \chi_{k_{s}}\left(\tau_{k_{s}}\right)}\left|p_{0}\right\rangle \prod_{i=1}^{s} d \tau_{i} .
\end{aligned}
$$

It follows from definition (19) that each term in this series corresponds to the function $Q_{s}^{\chi}\left(\left\{\tau_{i}, k_{i}, \sigma_{i}\right\}\right)$, namely,

$$
\begin{gathered}
Q_{0}=\left\langle q_{0}\left|e^{-\gamma T}\right| n_{s}\right\rangle \\
Q_{s}^{\chi}\left(\left\{\tau_{i}, k_{i}, \sigma_{i}\right\}\right)=\left\langle q_{0}\right| T_{\tau} \exp \left\{-\int_{-T / 2}^{T / 2} \hat{\gamma}(\tau) d \tau\right\} \hat{\Gamma}_{k_{1}}^{\left(\sigma_{1}\right)}\left(\tau_{1}\right) \\
\times e^{i \sigma_{1} \chi_{k_{1}}\left(\tau_{k_{1}}\right) \cdots \hat{\Gamma}_{k_{s}}^{\left(\sigma_{s}\right)}\left(\tau_{s}\right) e^{i \sigma_{s} \chi_{k_{s}}\left(\tau_{k_{s}}\right)}\left|n_{s}\right\rangle .}
\end{gathered}
$$

Therefore Eqs. (24) and (23) are reduced to previous result (20). This completes the proof. Note, that owing to the property Eq. (8), $Z_{0}=\left\langle q_{0}|\exp (-T \hat{L})| n_{s}\right\rangle=1$ identically at $\chi=0$. Therefore, probabilities (19) are correctly normalized.

Equation (23) for the generating function $Z\left[\left\{\chi_{i}(t)\right\}\right]$ depends on the initial-state $\left|n_{s}\right\rangle$ of the system. It can be shown that the choice of $\left|n_{s}\right\rangle$ does not affect the final results. We assume that $\chi_{k}(t) \rightarrow 0$ when $t \rightarrow-T / 2$. Physically, it means that the measurement is limited in time. To be specific one may assume that $\chi_{k}(t)=0$ when $-T / 2<t<-T / 2+\Delta t$ and $\chi_{k}(t) \neq 0$ if $t>-T / 2+\Delta t$. If the time interval $\Delta t$ is sufficiently large as compared with the typical transition time $\Gamma^{-1}$, then the system will reach the steady state during this period of time. The latter follows from the fact that $\exp (-\hat{L} \Delta t)\left|n_{s}\right\rangle \rightarrow\left|p_{0}\right\rangle$ when $\Delta t \gg \Gamma^{-1}$. Thus one can substitute $\left|n_{s}\right\rangle$ to $\left|p_{0}\right\rangle$ in Eq. (23). Assuming also the limit $T$ $\rightarrow \infty$, we arrive to the main result of this section

$$
\exp \left(-S\left[\left\{\chi_{i}(t)\right\}\right]\right)=\left\langle q_{0}\left|T_{\tau} \exp \left\{-\int_{-\infty}^{+\infty} \hat{L}_{\chi}(\tau) d \tau\right\}\right| p_{0}\right\rangle .
$$


We see that the generating function can be written in the form of the averaged evolution operator. This operator corresponds to master equation with the rates modified by the counting fields $\chi_{i}(\tau)$.

Further simplification is valid in the low-frequency limit of the current correlations, $\omega \ll \Gamma$. (Here $\Gamma$ is a typical transition rate in the system.) In this situation one can assume that the counting fields $\chi_{k}(t)$ are turned on and switched off adiabatically. Then, if $t_{0} \gg \Gamma^{-1}$ is the time of measurement, the action [Eq. (26)] reduces to

$$
S\left(\left\{\chi_{i}\right\}\right)=t_{0} \Lambda_{\min }\left(\left\{\chi_{i}\right\}\right),
$$

where $\Lambda_{\min }\left(\left\{\chi_{i}\right\}\right)$ is a minimal eigenvalue of the operator $\hat{L}_{\chi}$. As one can see the problem of statistics in question, provided the transition rates in the system are known, is merely a problem of the linear algebra. The probability $P\left(\left\{N_{i}\right\}\right)$ of $N_{i}$ electrons to be transferred through the corresponding terminal during the time $t_{0}$ is then expressed via $S\left(\left\{\chi_{i}\right\}\right)$ with the use of relation (18).

It is worth to mention that the another definition of FCS, different from that given by Eq. (27), is widely used in quantum optics. ${ }^{27-29}$ The definition of FCS in quantum optics makes use of the sudden turning on of counting fields $\chi_{k}(t)$ at time zero and subsequent abrupt switching them of after time $t_{0}$. Under this assumption the action (26) becomes

$$
\exp \left\{-S\left(\left\{\chi_{i}\right\}\right)\right\}=\left\langle q_{0} \mid p_{\chi}^{(n)}\right\rangle \exp \left(-t_{0} \Lambda_{n}\left(\left\{\chi_{i}\right\}\right)\left\langle q_{\chi}^{(n)} \mid p_{0}\right\rangle .\right.
$$

Here $\left\langle q_{\chi}^{(n)}\right|$ and $\left|p_{\chi}^{(n)}\right\rangle$ denote the eigenvectors of the $\hat{L}_{\chi}$ operator, and $\Lambda_{n}\left(\left\{\chi_{i}\right\}\right)$ is its spectrum. The probability $P\left(\left\{N_{i}\right\}, t_{0}\right)$ can be found as before via relation (18). Then so defined FCS can be used to find different two-point correlation functions of two photoelectrons. ${ }^{28,29}$ Note, that above relations holds at time scales $t_{0}$ of the order of average waiting time between two successive photocount events. Contrary to that, definition (27) of FCS, accepted in mesoscopics physics makes sense at time scale, which greatly exceeds the average time between two successive events of charge transfer.

Consider now the question of the current conservation in the nodes. For this purpose we associate the counting fields $\chi_{k}$ with each line $k=\{\alpha, \beta\}$ of the graph and in the appropriate way modify the $L_{\chi}$ operator. Then we define the classical current operator $\hat{J}_{\chi}^{(k)} \equiv \hat{J}_{\chi}^{\{\alpha, \beta\}}$ through each line by means of the relation

$$
\hat{J}_{\chi}^{(k)}=i e \frac{\partial \hat{L}_{\chi}}{\partial \chi_{k}} \equiv e\left(\hat{\Gamma}_{k}^{(+)} e^{i \chi_{k}-\hat{\Gamma}_{k}^{(-)}} e^{\left.-i \chi_{k}\right)} .\right.
$$

Its average value can be found via relation

$$
I_{k}\left(\left\{\chi_{i}\right\}\right)=\frac{i e}{t_{0}} \frac{\partial S}{\partial \chi_{k}}=\left\langle q_{\chi}^{(0)}\left|\hat{J}_{\chi}^{(k)}\right| p_{\chi}^{(0)}\right\rangle .
$$

It follows from the Eq. (27) and the fact that $\Lambda_{\min }\left(\left\{\chi_{i}\right\}\right)$ $=\left\langle q_{\chi}^{(0)}\left|\hat{L}_{\chi}\right| p_{\chi}^{(0)}\right\rangle$ with $\left\langle q_{\chi}^{(0)}\right|$ and $\left|p_{\chi}^{(0)}\right\rangle$ being the eigenvectors of the $\hat{L}_{\chi}$ operator.
The average physical currents for $k \leqslant N$ are given by $\bar{I}_{k}$ $=\left.I_{k}\left(\left\{\chi_{i}\right\}\right)\right|_{\chi=0}$. Expanding the vector notation in Eq. (29) one gets the usual relation for the current in the master equation method. The current is expressed via transition rates and the steady probability distribution $p_{0}(\{n\})$.

We also introduce the particle number operator $\hat{n}^{\{\alpha\}}$ in each node, given by a usual formula

$$
\hat{n}^{\{\alpha\}}=\sum_{\{n\}}|n\rangle n_{\alpha}\langle n| .
$$

Then after few algebra one see that the relation

$$
\sum_{\beta} \pm \hat{J}_{\chi}^{\{\alpha, \beta\}}=-e\left[\hat{n}^{\{\alpha\}}, \hat{L}_{\chi}\right]
$$

always holds at any node $\alpha$. Here the summation is going over all nodes $\beta$, connected to $\alpha$. The choice of the sign in front of each term under the sum depends on the situation whether the given directed line $k=\{\alpha, \beta\}$ is going out or coming into the chosen node $\alpha$. The Eq. (31) gives the charge conservation law in the operator language. Averaging the latter expression over the steady distribution, $\left\langle q_{0}|\cdots| p_{0}\right\rangle$, and using Eqs. (9) and (29) we arrive at the conservation law for the $\chi$-dependent currents at each node $\alpha$

$$
\sum_{\beta} \pm I_{\{\alpha, \beta\}}\left(\left\{\chi_{i}\right\}\right)=0 .
$$

This also ensures the conservation of the physical current in the model $\Sigma_{k} \bar{I}_{k}=0$, where the sum is extended only to the external junctions $k$. It follows from summing up relations (32) over all internal nodes $\alpha$ and setting $\chi=0$ afterwards.

\section{RESONANT-LEVEL MODEL}

In this section we consider the current statistics of the resonant-level model. First we focus on the noninteracting case. Then we apply the general result of the Sec. III to the strongly interacting case and compare the statistics in these two different regimes. In the end of the section we rederive the results of the previous works concerning the shot noise in these systems.

Following definition (21) and the expression for the rates Eq. (4), the $\hat{L}_{\chi}$-matrix of the single resonant-level model in the noninteracting regime reads as

$$
\hat{L}_{\chi}=\left(\begin{array}{cc}
\Gamma_{1 \leftarrow 0} & -\Gamma_{0 \leftarrow 1}(\chi) \\
-\Gamma_{1 \leftarrow 0}(\chi) & \Gamma_{0 \leftarrow 1}
\end{array}\right),
$$

where

$$
\begin{gathered}
\Gamma_{1 \leftarrow 0}(\chi)=\Gamma_{L} f_{L} e^{-i \chi_{1}}+\Gamma_{R} f_{R} e^{-i \chi_{2}}, \\
\Gamma_{0 \leftarrow 1}(\chi)=\Gamma_{L}\left(1-f_{L}\right) e^{i \chi_{1}}+\Gamma_{R}\left(1-f_{R}\right) e^{i \chi_{2}} .
\end{gathered}
$$

Evaluating the minimal eigenvalue of this matrix one obtains the current statistics in the form 


$$
\begin{gathered}
S(\chi)=t_{0}\left\{\Gamma_{L}+\Gamma_{R}-\sqrt{\mathcal{D}(\chi)}\right\}, \\
\mathcal{D}(\chi)=\left(\Gamma_{L}+\Gamma_{R}\right)^{2}+4 \Gamma_{L} \Gamma_{R}\left[f_{(-)}\left(\epsilon_{i}\right)\left(e^{-i \chi}-1\right)\right. \\
\left.+f_{(+)}\left(\epsilon_{i}\right)\left(e^{i \chi}-1\right)\right],
\end{gathered}
$$

Here $f_{(-)}\left(\epsilon_{i}\right)=f_{L}\left(\epsilon_{i}\right)\left[1-f_{R}\left(\epsilon_{i}\right)\right], \quad f_{(+)}\left(\epsilon_{i}\right)=f_{R}\left(\epsilon_{i}\right)[1$ $\left.-f_{L}\left(\epsilon_{i}\right)\right]$, and $\chi=\chi_{1}-\chi_{2}$. We have also accounted for the double occupancy of the level by multiplying the result by two.

Since the Coulomb blockade phenomenon is completely disregarded in this model, one might have come to the same result in the framework of the pioneering approach by Levitov and co-workers. ${ }^{2}$ We will show now that it is indeed the case.

According to Ref. 2 the general expression for the current statistics through a single contact is written as

$$
\begin{aligned}
S(\chi)= & -\frac{t_{0}}{\pi} \sum_{n} \int d \epsilon \ln \left\{1+T_{n}(\epsilon)\left(f _ { L } ( \epsilon ) [ 1 - f _ { R } ( \epsilon ) ] \left(e^{-i \chi}\right.\right.\right. \\
& \left.\left.-1)+f_{R}(\epsilon)\left[1-f_{L}(\epsilon)\right]\left(e^{i \chi}-1\right)\right)\right\} .
\end{aligned}
$$

It is valid for any two-terminal geometry provided the region in between two electrodes can be described by the oneparticle scattering approach and the effects of interaction are of no importance. $T_{n}(\epsilon)$ is a set of transmission eigenvalues that are in general energy-dependent. Fermi functions include the effects of applied voltage and the temperature. For a single resonant level there is a single resonant transmission eigenvalue $T_{r}(\epsilon)$, its energy dependence being given by the Breit-Wigner formula

$$
T_{r}(\epsilon)=\frac{\Gamma_{L} \Gamma_{R}}{\left(\epsilon-\epsilon_{i}\right)^{2}+\left(\Gamma_{L}+\Gamma_{R}\right)^{2} / 4} .
$$

Here $\epsilon_{i}$ denotes a position of a resonant level. The result Eq. (36) is more general, than Eq. (35). When electrons do not interact Eq. (36) is valid for any temperature. We will show below that one can reproduce the statistics Eq. (35) on substituting $T_{r}(\epsilon)$ into Eq. (36) and assuming the regime $k_{B} T$ $\gg \hbar \Gamma$. As it was discussed previously, this is the condition, when the master equation approach, and hence its consequence Eq. (35), are valid.

It is easier to perform the calculation if one first evaluates the $\chi$-dependent current $I(\chi)=\left(i e / t_{0}\right) \partial S / \partial \chi$. It reads

$$
\begin{aligned}
I(\chi)= & \frac{1}{\pi} \int d \epsilon\left[f_{(+)}(\epsilon) e^{i \chi}-f_{(-)}(\epsilon) e^{-i \chi}\right]\left\{T_{r}^{-1}(\epsilon)\right. \\
& \left.+\left[f_{(-)}(\epsilon)\left(e^{-i \chi}-1\right)+f_{(+)}(\epsilon)\left(e^{i \chi}-1\right)\right]\right\} .
\end{aligned}
$$

In what follows we assume that the resonant level is placed between the chemical potentials $\mu_{L\{R\}}$ in the leads. Since $k_{B} T \gg \Gamma_{L(R)}$, the main contribution comes from the Lorentz peak and one can put $\epsilon=\epsilon_{i}$ in the Fermi functions. Therefore, we left only with the two poles $\epsilon_{1(2)}=\epsilon_{i} \pm i \sqrt{\mathcal{D}(\chi)} / 2$ under the integrand Eq. (38). Closing the integration contour in the upper or lower half plane we arrive at

$$
I(\chi)=2 e \Gamma_{L} \Gamma_{R}\left[f_{(+)}\left(\epsilon_{i}\right) e^{i \chi}-f_{(-)}\left(\epsilon_{i}\right) e^{-i \chi}\right] / \sqrt{\mathcal{D}(\chi)} .
$$

On integrating it over $\chi$ one finds for the $S(\chi)$ $=\left(t_{0} /\right.$ ie $) \int_{0}^{\chi} I\left(\chi^{\prime}\right) d \chi^{\prime}$ the result Eq. (35) obtained by means of master equation. Thus, we have verified the correspondence between two approaches to statistics in the noninteracting regime.

To proceed we address the strongly interacting regime. In this case the $(2 \times 2) \hat{L}_{\chi}$-matrix is formed with the use of rates Eq. (3). It has a structure similar to Eq. (34), provided the $\chi$-dependent rates are written as

$$
\begin{gathered}
\Gamma_{1 \leftarrow 0}(\chi)=2 \Gamma_{L} f_{L} e^{-i \chi_{1}}+2 \Gamma_{R} f_{R} e^{-i \chi_{2}}, \\
\Gamma_{0 \leftarrow 1}(\chi)=\Gamma_{L}\left(1-f_{L}\right) e^{i \chi_{1}}+\Gamma_{R}\left(1-f_{R}\right) e^{i \chi_{2}} .
\end{gathered}
$$

Evaluating the corresponding eigenvalue $\hat{L}_{\chi}$ one can write down the expression for statistics in the strongly interacting limit

$$
\begin{aligned}
S(\chi)= & \left(t_{0} / 2\right)\left\{\Gamma_{L}\left[1+f_{L}\left(\epsilon_{i}\right)\right]+\Gamma_{R}\left[1+f_{R}\left(\epsilon_{i}\right)\right]-\sqrt{\mathcal{D}(\chi)}\right\}, \\
\mathcal{D}(\chi)= & \left\{\Gamma_{L}\left[1+f_{L}\left(\epsilon_{i}\right)\right]+\Gamma_{R}\left[1+f_{R}\left(\epsilon_{i}\right)\right]\right\}^{2} \\
& +8 \Gamma_{L} \Gamma_{R}\left[f_{(-)}\left(\epsilon_{i}\right)\left(e^{i \chi}-1\right)+f_{(+)}\left(\epsilon_{i}\right)\left(e^{-i \chi}-1\right)\right] .
\end{aligned}
$$

To proceed we consider the shot-noise regime $e V \gg k_{B} T$ and assume that the voltage is applied to the right electrode as shown in Fig. 2. Then the temperature fluctuations become nonessential and both statistics (35) and (40) take a rather simple form

$$
\left.S(\chi)\right|_{U=0}=t_{0}\left\{\Gamma_{L}+\Gamma_{R}-\sqrt{\left(\Gamma_{R}-\Gamma_{L}\right)^{2}+4 \Gamma_{L} \Gamma_{R} e^{-i \chi}}\right\},
$$

$$
\left.S(\chi)\right|_{U \rightarrow \infty}=\frac{1}{2} t_{0}\left\{2 \Gamma_{L}+\Gamma_{R}-\sqrt{\left(2 \Gamma_{L}-\Gamma_{R}\right)^{2}+8 \Gamma_{L} \Gamma_{R} e^{-i \chi}}\right\} .
$$

Given the latter expressions at hand one can rederive the known result for the average current $I=\left(i e / t_{0}\right) \partial S /\left.\partial \chi\right|_{\chi}=0$ and the shot-noise power $S_{\text {shot }}=\left(e^{2} / t_{0}\right) \partial^{2} S /\left.\partial \chi^{2}\right|_{\chi=0}$ in these models. It is conventional to represent $S_{\text {shot }}$ in the form $S_{\text {shot }}=e I F$, where $F$ is the so-called Fano factor. Its importance to the mesocscopic transport has been pointed out in the work. ${ }^{30}$ Its deviation from the unity indicates to the nonPoissonian type of electron counting statistics. The latter fact, in its turn, reveals the presence of correlations of the successive electron transfer. For the single resonant-level model one obtains $F=\left(\Gamma_{L}^{2}+\Gamma_{R}^{2}\right) /\left(\Gamma_{L}+\Gamma_{R}\right)^{2}$ in the noninteracting regime and $F=\left(\Gamma_{L}^{2}+4 \Gamma_{R}^{2}\right) /\left(\Gamma_{L}+2 \Gamma_{R}\right)^{2}$ in the Coulomb blockade limit. ${ }^{26}$

As one can see from the Eq. (41), at low temperatures, the difference of statistics in the large $U$ limit from the one in the noninteracting case is an effective suppression of $\Gamma_{R}$ rate by a factor of two. To find the probability distribution $P\left(N, t_{0}\right)$ one can estimate the integral Eq. (18) by means of steepest descent method. It is applicable in the given case of lowfrequency regime $\omega \ll \Gamma$, which we consider, since both the action $S(\chi) \gg 1$ and the average number of transmitted electrons $\bar{N}=\bar{I} t_{0} / e \gg 1$. Then one has to find the saddle-point $\chi_{*}$ of the function $\Omega(\chi)=S(\chi)+i \chi I t_{0} / e$, which is defined by 


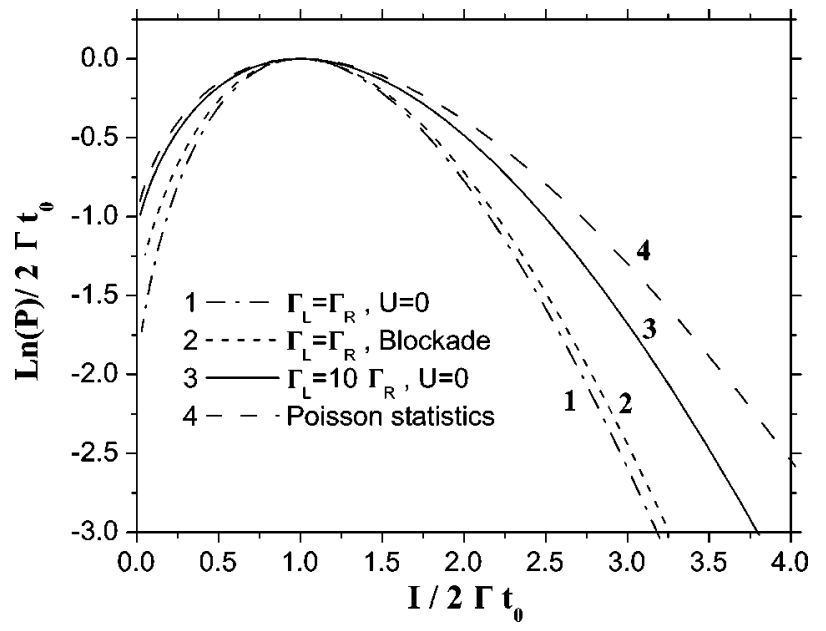

FIG. 4. The current statistics through the single resonant level. 1 and 4 , noninteracting model; 2 , interacting model; 4 , Poisson statistics.

the equation $I=\left(i e / t_{0}\right)[\partial S / \partial \chi]$. It is turned out that $\chi_{*}$ always lies on the imaginary axis. This equation can be regarded as a parametric relation between $I$ and $\chi_{*}$, and with the exponential accuracy we obtain the estimation for the probability $P(I) \sim \exp \left[-\Omega\left(\chi_{*}\right)\right]$.

The results for statistics (41) are shown in Fig. 4. The statistics are compared with the Poisson-type statistics $S(\chi)$ $=2 \Gamma t_{0}[\exp (i \chi)-1]$ with the effective rate given by $\Gamma^{-1}$ $=\Gamma_{L}^{-1}+\Gamma_{R}^{-1}$. Both statistics (41) approach the Poisson one, provided the system is strongly asymmetric, $\Gamma_{L} \gg \Gamma_{R}$.

\section{THE FCS IN THE COULOMB BLOCKADE QUANTUM DOTS}

In this section we discuss the application of the method to the many-terminal Coulomb blockade island. The consideration will be limited to the two- and three-terminal layouts. Our treatment will be mainly numerical, though some analytical results in the two-terminal setup are also plausible. In the beginning of the Sec. a few technical details, which are common for both cases, are given. In particular, we establish the relation of the FCS approach with the preceding papers, concerning the shot noise in the conventional single electron transistor. In the following we consider the FCS, first for two-terminal, and then for three-terminal configurations. We will also compare the FCS in the strongly Coulomb blockade limit with our recent results, concerning the FCS in manyterminal chaotic quantum dot with contacts being the tunnel junctions.

\section{A. General remarks}

In the framework of the "orthodox theory" the macroscopic state of Coulomb blockade island is characterized by the excess charge $Q=n e$, which is quantized in terms of electron charge $(-e)$. This charge $Q$ can be changed only by $\pm e$ in course of one tunneling event. Therefore, the master equation connects the given macroscopic state $n$ with only the neighboring states $n \pm 1$. The corresponding rates
$\Gamma_{n \pm 1 \leftarrow n}$ of these transitions are equal to the sum of $N=2$ or 3 independent probabilities $\Gamma_{n \pm 1 \leftarrow n}^{(k)}$ through the different junctions, those are given by Eqs. (5) and (6). Along with the lines of Sec. III we have to modify the rates $\Gamma_{n \pm 1 \leftarrow n}$ into $\chi$-dependent quantities $\Gamma_{n \pm 1 \leftarrow n}^{\chi}$ in accordance with the rule Eq. (21). After that, in order to find the FCS of the charge transfer through the island, one has to evaluate the minimal eigenvalue $\Lambda_{\min }$ of the three-diagonal matrix $\hat{L}_{\chi}$. In the given case it is convenient to write down the latter problem as the eigenvalue problem for the following set of linear equations

$$
\left(\Lambda-\gamma_{n}\right) p_{n}+\Gamma_{n \leftarrow n+1}^{\chi} p_{n+1}+\Gamma_{n \leftarrow n-1}^{\chi} p_{n-1}=0,
$$

where $\quad \gamma_{n}=\Gamma_{n \leftarrow n-1}+\Gamma_{n \leftarrow n+1}, \quad$ and $\quad \Gamma_{n \leftarrow n \pm 1}^{\chi}$ $=\sum_{k=1}^{N} \Gamma_{n \pm 1 \leftarrow n}^{(k)} e^{ \pm i \chi_{k}}$. The index $+(-)$ corresponds to electron transition from (to) the island.

In general, we have treated the problem Eq. (43) numerically. At sufficiently low temperatures $k_{B} T \ll E_{c}$, which is mainly the case of the following discussion, the temperature dependence in rates Eq. (5) is nonessential. Then one can set $\Gamma_{k}^{( \pm)}(n)=\Delta E_{n \pm 1 \leftarrow n}^{(k)} /\left(e^{2} R_{k}\right)$ when $\Delta E_{n \pm 1 \leftarrow n}^{(k)} /\left(e^{2} R_{k}\right) \geqslant 0$ and $\Gamma_{k}^{( \pm)}(n)=0$ otherwise. Thus defined rates are linear functions in $n$. The possible set of $\{n\}$, corresponding to nonvanishing rates, is limited to some interval $n_{\text {min }} \leqslant n \leqslant n_{\text {max }}$. Hence Eq. (43) becomes a finite linear problem. At higher temperatures $k_{B} T \leqslant E_{c}$ we have found that the increase (decrease) of both $n_{\min }$ and $n_{\max }$ to extra $7 / 8$ states gives the results up to $10^{-15}$ degree of accuracy in course of the numerical procedure.

The matrix $\hat{L}_{\chi}$ of Eq. (43) is non-Hermitian. This fact may cause an instability in the numerical algorithm when the range $\left[n_{\min }, n_{\max }\right]$ is large. However, in most practical cases, this problem can be circumvented by transforming $\hat{L}_{\chi}$ to Hermitian form. First we note, that one only needs to work with pure imaginary counting fields $\chi_{k}$, as long as the probability $P\left(\left\{N_{i}\right\}, t_{0}\right)$ is estimated in the saddle-point approximation. (See the discussion in the end of the Sec. III.) Hence the rates $\Gamma_{n \leftarrow n \pm 1}^{\chi}$ become the positive real numbers. Then we can apply the linear transformation $p_{n}^{\prime}=A_{n} p_{n}$. It leads to the rates in the new gauge $\Gamma_{n \leftarrow n \pm 1}^{\prime \chi}=A_{n+1} \Gamma_{n \leftarrow n \pm 1}^{\chi} A_{n}^{-1}$. The unknown $A_{n}$ 's may be chosen in a way that the symmetry relation $\Gamma_{n \leftarrow n \pm 1}^{\prime \chi}=\Gamma_{n \pm 1 \leftarrow n}^{\prime \chi}$ would hold. This gives the recurrent relation $A_{n+1} / A_{n}=\left(\Gamma_{n \leftarrow n+1}^{\chi} / \Gamma_{n+1 \leftarrow n}^{\chi}\right)^{1 / 2}$. With the use of latter the Eq. (43) takes the Hermitian form when it is written in terms of $p_{n}^{\prime}$ and transformed rates $\Gamma_{n \leftarrow n \pm 1}^{\prime \chi}$ $=\left(\Gamma_{n \leftarrow n \pm 1}^{\chi} \Gamma_{n \pm 1 \leftarrow n}^{\chi}\right)^{1 / 2}$. The diagonal term $\gamma_{n}$ is not affected under this transformation.

Let us also discuss a useful relation for the shot-noise correlations $S_{k m}=\left(e^{2} / t_{0}\right) \partial^{2} S /\left.\partial \chi_{k} \partial \chi_{m}\right|_{\chi=0}$. One can use the identity $\Lambda_{\min }\left(\left\{\chi_{i}\right\}\right)=\left\langle q_{\chi}^{(0)}\left|\hat{L}_{\chi}\right| p_{\chi}^{(0)}\right\rangle$ in order to express them in terms of eigenvectors $\left|p_{n}\right\rangle,\left\langle q_{n}\right|$ and eigenvalues $\lambda_{n}$ of the matrix $\hat{L}$. With the use of standard algebra and assuming the normalization $\left\langle q_{n} \mid p_{n^{\prime}}\right\rangle=\delta_{n, n^{\prime}}$ we can cast $S_{k m}$ in the form 


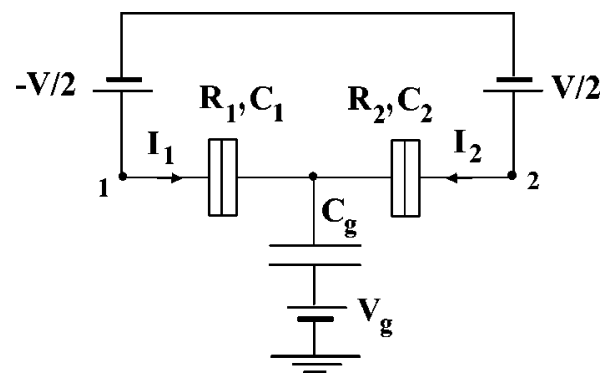

FIG. 5. The equivalent circuit of the two-terminal Coulomb blockade island.

$$
\begin{aligned}
S_{k m}= & e^{2}\left\langle q_{0}\left|\hat{S}^{(k, m)}\right| p_{0}\right\rangle+e^{2} \sum_{l>0} \frac{1}{\lambda_{l}}\left\{\left\langle q_{0}\left|\hat{J}_{k}\right| p_{l}\right\rangle\left\langle q_{l}\left|\hat{J}_{l}\right| p_{0}\right\rangle\right. \\
& \left.+\left\langle q_{0}\left|\hat{J}_{m}\right| p_{l}\right\rangle\left\langle q_{l}\left|\hat{J}_{k}\right| p_{0}\right\rangle\right\}
\end{aligned}
$$

where the $\hat{J}_{k}$ operator was defined by Eq. (28) and $\hat{S}^{(k, m)}$ $=\partial^{2} \hat{L}_{\chi} /\left.\partial \chi_{k} \partial \chi_{m}\right|_{\chi=0}$. Note, that relation (44) holds in any basis. One may, in particular, use it for the basis $p_{n}^{\prime}$ discussed above. In this case one must define the matrix elements of $\hat{J}_{k}$ as $\left[\hat{J}_{k}\right]_{n \pm 1, n}=\left(i \partial / \partial \chi_{k}\right) \Gamma_{n \leftarrow n \pm 1}^{\prime \chi}$ and to evaluate $\hat{S}^{(k, m)}$ in the same manner.

It follows from relation (44) that the shot-noise correlations are defined by the whole spectrum of the relaxationtimes $\tau_{k}^{-1}=\lambda_{k}$ in the system. In case of two-terminal geometry it coincides with preceding results of Refs. 14 and 15.

\section{B. Two-terminal Coulomb blockade island}

The electrical circuit with a two-terminal Coulomb blockade island is shown in Fig. 5. The dot is biased in such a way, that $V_{2}=-V_{1}=V / 2$. At low-temperatures $k_{B} T$ $\ll e^{2} / C_{\Sigma}$ the $\chi$-dependent rates $\Gamma_{n \leftarrow n \pm 1}^{\chi}$ reads as

$$
\begin{aligned}
& \Gamma_{n+1 \leftarrow n}^{\chi}=\left[\frac{\widetilde{C}_{1} V}{e}-\left(n+\frac{C_{g} V_{g}}{e}+\frac{1}{2}\right)\right] \frac{e^{i \chi_{2}}}{R_{2} C_{\Sigma}}, \\
& \Gamma_{n-1 \leftarrow n}^{\chi}=\left[\frac{\widetilde{C}_{2} V}{e}+\left(n+\frac{C_{g} V_{g}}{e}-\frac{1}{2}\right)\right] \frac{e^{-i \chi_{1}}}{R_{1} C_{\Sigma}},
\end{aligned}
$$

where $\widetilde{C}_{1(2)}=C_{1(2)}+C_{g} / 2$ are effective capacitances. The gate voltage $V_{g}$ can be used to control the offset charge $q_{0}$ $=C_{g} V_{g}$ on the dot. It can be varied continuously in the range $-e / 2 \leqslant q_{0} \leqslant e / 2$. The resulting dimension of the matrix $\hat{L}_{\chi}$ is given by the number of absorbing states $n_{\max }-n_{\min }$, where $n_{\min }\left(n_{\max }\right)$ is the maximal (minimal) integer closest to the points $n_{1,2}$ where the rates $\Gamma_{n \mp 1 \leftarrow n}^{\chi}$ vanish.

First, we briefly consider the voltage dependence of the shot noise in the system. ${ }^{14,15}$ It was calculated with the use of Eq. (44). The results for the noise-to-current ratio (Fano factor) are presented in Figs. 6-8. The Coulomb blockade features are strongly pronounced in case of asymmetric junctions only. The resistances of the contacts are much easier to vary than the mutual capacitances in the experimental situation, when the dot is made up with the use of 2D-electron gas in the semiconducting heterostructure. Therefore, we have

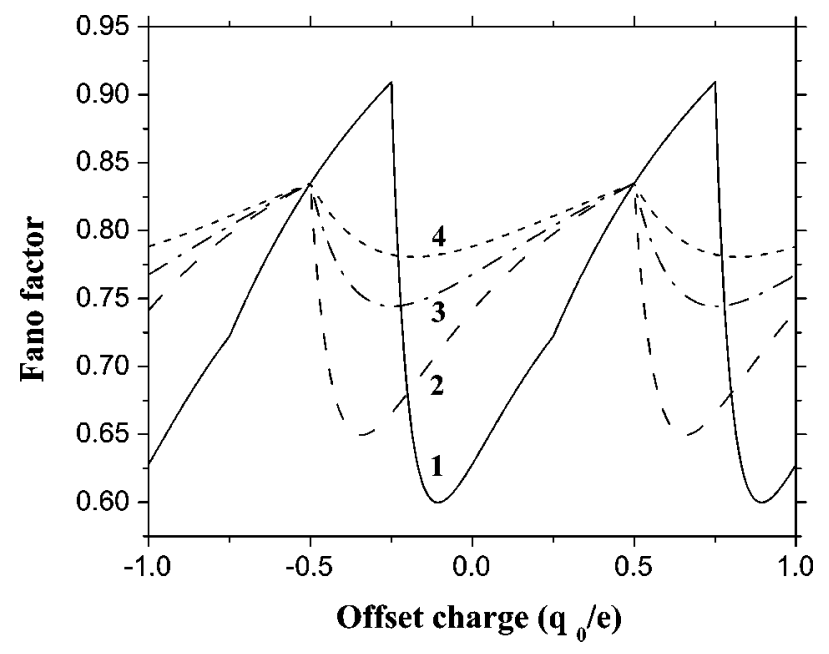

FIG. 6. The Fano factor versus the offset charge in the twoterminal Coulomb blockade island. The parameters are: $C_{1}=C_{2}$, $R_{1}=10 R_{2}, T=0.01 e^{2} / 2 C_{\Sigma}$. (1) $V=1.5 e / C_{\Sigma}$, (2) $V=2.0 e / C_{\Sigma}$, (3) $V=4.0 e / C_{\Sigma}$, (4) $V=6.0 e / C_{\Sigma}$.

chosen $\widetilde{C}_{1}=\widetilde{C}_{2}$ and plotted the Fano factor for different values of ratio $R_{2} / R_{1}$ and offset charge $q_{0}$. The curves are truncated below the Coulomb blockade threshold, where the considered "orthodox" theory is not applicable. At high values of the ratio $R_{2} / R_{1}$ they exhibit the strong characteristic Coulomb blockade oscillations. The special points at the voltage dependences occur when either $n_{\min }$ or $n_{\max }$ are changed by 1 . At high bias voltages the noise-to-current ratio saturates to the value $F=\left(R_{1}^{2}+R_{2}^{2}\right) /\left(R_{1}+R_{2}\right)^{2}$ independently of the capacitances $C_{k}$ and the offset charge $q_{0}$. (See the discussion below as well.) An increase of a temperature leads to the smearing of oscillations due to the additional thermal noise. The above results coincide with those, obtained previously by Hershfiled et al. ${ }^{15}$

To proceed we turn to the question of the FCS. For the sake of clarity we first present our recent analytical results

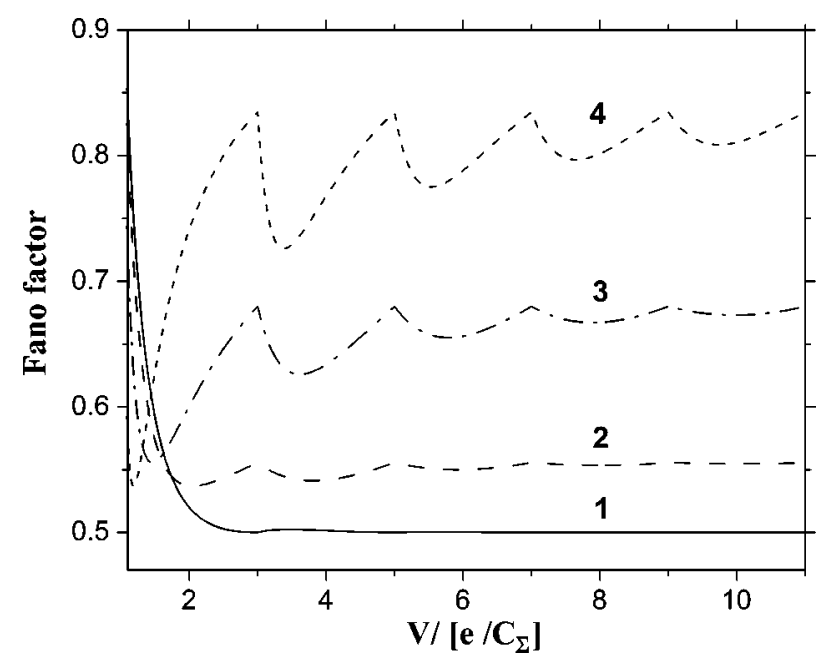

FIG. 7. The Fano factor versus the applied voltage in the twoterminal Coulomb blockade island. The parameters are: $C_{1}=C_{2}$, $k_{B} T \ll e^{2} / 2 C_{\Sigma}, q_{0}=0$. (1) $R_{1} / R_{2}=1$, (2) $R_{1} / R_{2}=2$, (3) $R_{1} / R_{2}$ $=4$, (4) $R_{1} / R_{2}=10$. 


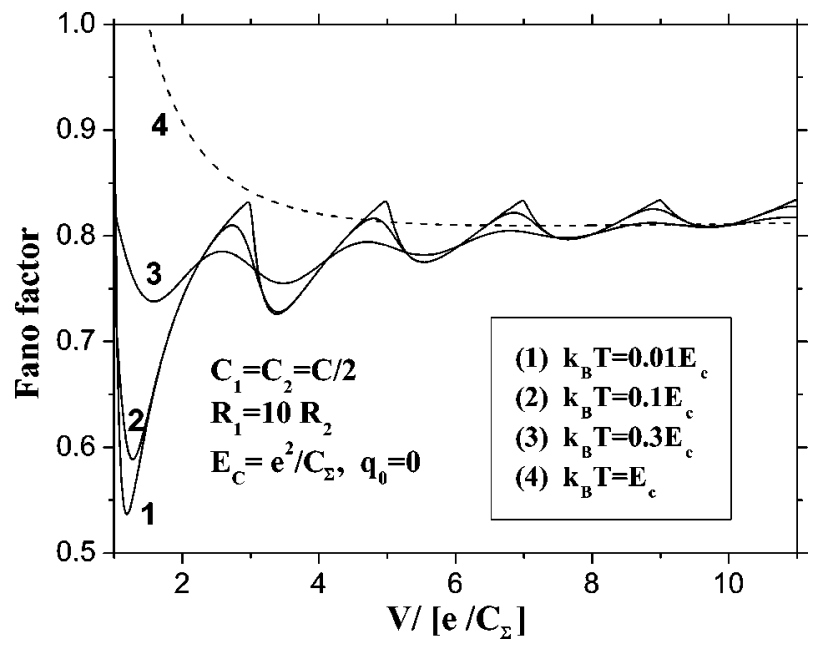

FIG. 8. The Fano factor versus the applied voltage at different temperatures. Parameters are shown on the plot. Temperature is given in terms of charging energy.

for the FCS of the chaotic quantum dot with two tunnel junctions, when their resistances $R_{k} \ll \pi \hbar / e^{2}$. In this case the effects of Coulomb interaction are negligible. Then we trace the differences in the FCS, when the dot is placed in the strongly interacting regime $R_{k} \gg \pi \hbar / e^{2}$.

In the noninteracting limit the action $S(\chi)$ is expressed via the voltage $V$ and the resistances $R_{k}$ only. ${ }^{11}$ At lowtemperatures $k_{B} T \ll e V$ it has a form similar to Eq. (41)

$$
\begin{aligned}
S(\chi)= & \frac{V t_{0}}{2 e}\left\{R_{1}^{-1}+R_{2}^{-1}\right. \\
& \left.-\sqrt{\left(R_{1}^{-1}-R_{2}^{-1}\right)^{2}+4\left(R_{1} R_{2}\right)^{-1} e^{i\left(\chi_{2}-\chi_{1}\right)}}\right\}
\end{aligned}
$$

It would be completely equivalent to the statistics of the charge transfer by noninteracting particles through the resonant level if one regards the ratios $\Gamma_{L, R}=V /\left(e R_{1,2}\right)$ as the effective tunneling rates. The generating function Eq. (46) gives the above mentioned value $F=\left(R_{1}^{2}+R_{2}^{2}\right) /\left(R_{1}+R_{2}\right)^{2}$ for the Fano factor.

In the strongly Coulomb blockade limit the action $S(\chi)$, in general, remarkably deviates from Eq. (46). Still, there are two exceptions, when $S(\chi)$ resembles the statistics (41) and (46).

The first case occurs at low voltages, slightly above the Coulomb blockade threshold value, when only one charging state is available for tunneling. This situation can be easily realized in the asymmetric dot with $R_{2} \neq R_{1}$. Then mere two states with $n=0$ and $n=1$ are involved and $\hat{L}_{\chi}$ is reduced to the $2 \times 2$ matrix Eq. (33). The only difference is that the rates $\Gamma_{L, R}$ contain the voltage dependence as given by Eq. (45). Thus the action $S(\chi)$ reproduces the result Eq. (41), where the rates $\Gamma_{L, R}$ are assumed to be voltage dependent.

To proceed we describe the second exceptional situation when the action $S(\chi)$ can be found analytically. Let $n_{1,2}$ be zeros of rates (45), i.e., $\Gamma_{n \pm 1 \leftarrow n}^{\chi}\left(n_{1,2}\right)=0$. We now interested in the situation when both zeroes $n_{1,2}$ simultaneously become integers. This situation may occur at the limited number of

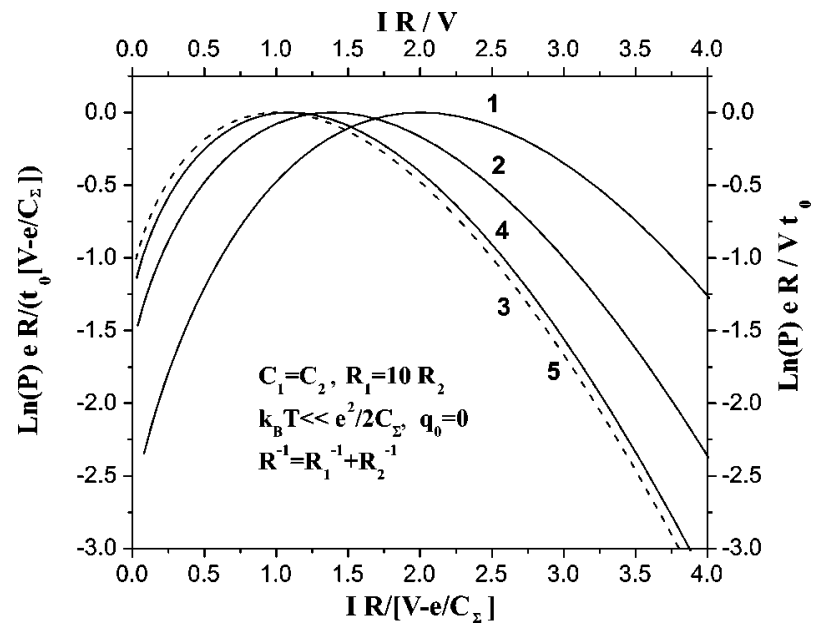

FIG. 9. The current statistics in the two-terminal quantum dot. Parameters are shown on the plot. Curves 3 and 5 coincide but correspond to different axes. (1) $V=1.5 e / C_{\Sigma}$, (2) $V=2.0 \mathrm{e} / C_{\Sigma}$, (3) $V=3.0 \mathrm{e} / C_{\Sigma}$, (4) $V=4.0 \mathrm{e} / C_{\Sigma}$, (5) noninteracting regime.

special points $V_{k}$ at the Coulomb blockade staircase when the ratio $\widetilde{C}_{1} / \widetilde{C}_{2}$ is close to a rational along with the special choice of the offset charge $q_{0}$. (E.g., for the configuration $\widetilde{C}_{1}=\widetilde{C}_{2}$, shown in Figs. 6 and 8, this is the case when (i) $q_{0}=0, \quad V_{k}=(2 k+1) e / C_{\Sigma} \quad$ and (ii) $q_{0}= \pm e / 2, \quad V_{k}$ $=2 k\left(e / C_{\Sigma}\right)$ with $k$ being integer.) In this situation we may show analytically (see Appendix B for the proof), that the action $S(\chi)$ at points $V_{k}$ takes the form similar to the statistics in the noninteracting regime (46). The only difference is that the voltage $V$ has to be substituted to $\left(\left|V_{k}\right|-e / C_{\Sigma}\right)$. The reduction of $V$ by the amount of the threshold voltage value $e / C_{\Sigma}$ is thus the manifestation of the Coulomb interaction. The given statistics is also valid as a limit at high voltages $V \gg e / C_{\Sigma}$. One may conclude it from the physically reasonable arguments that the result for the action in this limit should be linear function in voltage and must not depend on the capacitance ratio $\widetilde{C}_{1} / \widetilde{C}_{2}$. Hence, the statistics is insensitive to the fact, whether $n_{1,2}$ are integers or not. This also explains the saturation of the Fano factor at Figs. 6-8 to the noninteracting current-to-noise ratio.

To access the general situation, the whole problem has been treated numerically. We have evaluated the probability Eq. (18) in the saddle-point approximation along with the same lines as it was done for the resonant-level model. The Eq. (29) was used as the parametric relation between the current $I$ and the counting field $\chi=\chi_{2}-\chi_{1}$ in the saddlepoint $\chi_{*}$. In Fig. 9 we give the example of the logarithm of probability distribution $P(I)$ for the number of different voltages $V$ and offset charge $q_{0}=0$. All curves are normalized by the reduced value of the voltage $\left(V-e / C_{\Sigma}\right)$. For the value $V=3 e / C_{\Sigma}$ the renormalized logarithm of probability coincides with the one, obtained from the noninteracting limit (46). We have also plotted the same statistics in Fig. 4 in the resonant-level model for the ratio of rates $\Gamma_{L} / \Gamma_{R}=10$, when the interaction effects are disregarded. We see, that in general the probability distribution is strongly affected by the Coulomb blockade phenomenon, as compared to the noninteract- 


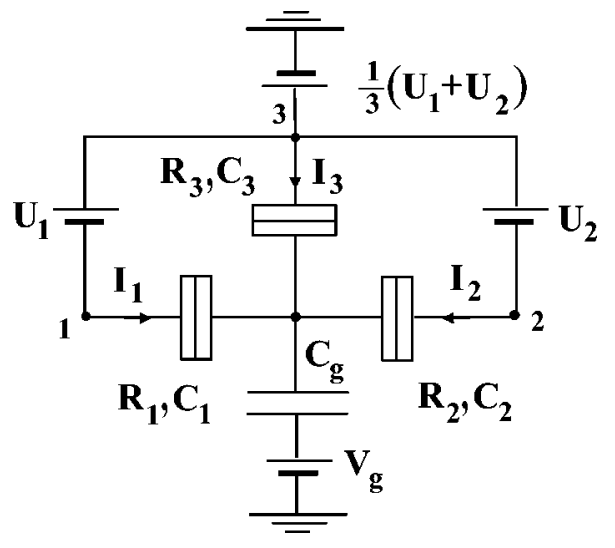

FIG. 10. The equivalent circuit of the three-terminal Coulomb blockade island. The voltages $U_{1(2)}$ are used to control the bias between the third and the first (the second) terminals, $U_{1(2)}=V_{3}$ $-V_{1(2)}$. The third terminal is biased at voltage $V_{3}=\left(U_{1}+U_{2}\right) / 3$ with respect to the ground. This setup assures the condition $V_{1}$ $+V_{2}+V_{3}=0$. Then gate voltage $V_{g}$ can be used to control the offset charge $q_{0}=C_{g} V_{g}$ on the island.

ing regime. It approaches to the noninteracting limit only at rather high-voltages $V \gg e / C_{\Sigma}$.

\section{Three-terminal Coulomb blockade island}

The electrical circuit with a three-terminal Coulomb blockade island is presented in Fig. 10. It is biased by three external voltage sources so that the current, flowing through the third terminal, would split into the first and the second ones. As in the preceding section we discuss the only lowtemperature regime $k_{B} T \ll e^{2} / C_{\Sigma}$. In what follows it is assumed that $U_{2}>U_{1}$. Then, according to Eqs. (5)-(7) and (21) the $\chi$-dependent rates of the system are written as follows:

$$
\begin{gathered}
\Gamma_{n+1 \leftarrow n}^{\chi}=\Gamma_{3}^{(+)}(n) e^{i \chi_{3}}+\Gamma_{1}^{(+)}(n) \theta(m-1 / 2-n) e^{i \chi_{1}}, \\
\Gamma_{n-1 \leftarrow n}^{\chi}=\Gamma_{2}^{(-)}(n) e^{-i \chi_{2}+\Gamma_{1}^{(-)}(n) \theta(n-m-1 / 2) e^{-i \chi_{1}},}
\end{gathered}
$$

where

$$
\Gamma_{k}^{( \pm)}(n)=a_{k}^{( \pm)} \mp\left(n+\frac{C_{g} V_{g}}{e} \pm \frac{1}{2}\right) \frac{1}{R_{k} C_{\Sigma}}
$$

and

$$
\begin{gathered}
a_{3}^{(+)}=\frac{\widetilde{C}_{1} U_{1}+\widetilde{C}_{2} U_{2}}{e R_{3} C_{\Sigma}}, \quad a_{2}^{(-)}=\frac{\left(\widetilde{C}_{1}+\widetilde{C}_{3}\right) U_{2}-\widetilde{C}_{1} U_{1}}{e R_{2} C_{\Sigma}}, \\
a_{1}^{( \pm)}= \pm \frac{\widetilde{C}_{2} U_{2}-\left(\widetilde{C}_{3}+\widetilde{C}_{2}\right) U_{1}}{e R_{1} C_{\Sigma}} .
\end{gathered}
$$

The effective capacitances $C_{k}$ are defined as $\widetilde{C}_{k}=C_{k}+C_{g} / 3$ and the point $q$ is determined by the relation

$$
q\left(U_{1}, U_{2}, V_{g}\right)=\frac{1}{e}\left\{\widetilde{C}_{2} U_{2}-\left(\widetilde{C}_{3}+\widetilde{C}_{2}\right) U_{1}-C_{g} V_{g}\right\} .
$$

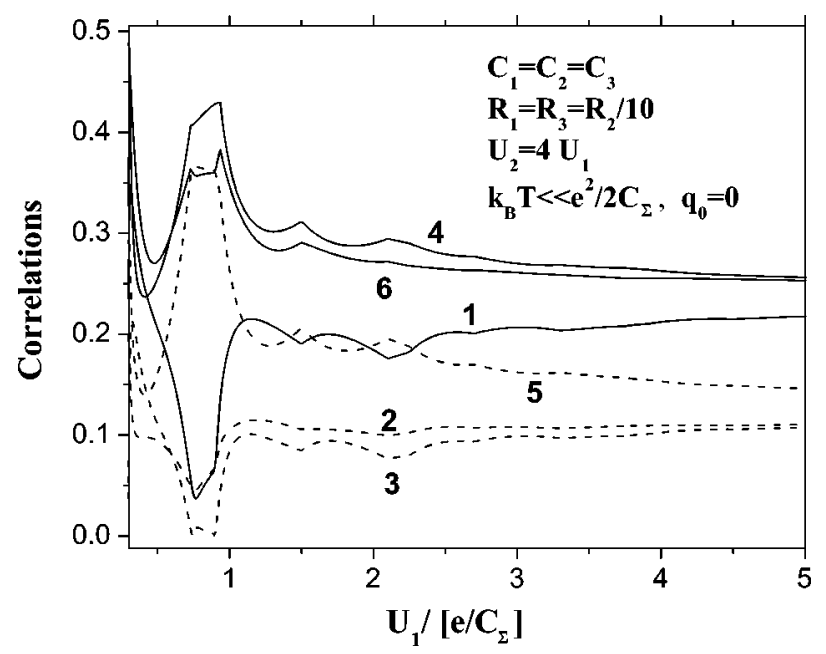

FIG. 11. The matrix $F$ of auto- and cross-shot-noise correlation versus voltage $U_{1}$ for the three-terminal quantum dot setup. Parameters are shown on the plot. (1) $F_{11}$, (2) $\left|F_{12}\right|$, (3) $\left|F_{13}\right|$, (4) $F_{22}$, (5) $\left|F_{23}\right|$, (6) $F_{33}$.

The value $q$ is noninteger in general. It satisfies the condition $\Gamma_{1}^{(-)}(q+1 / 2)=\Gamma_{1}^{(+)}(q-1 / 2)=0$. The dimension of the $\hat{L}_{\chi}$ matrix is equal to $n_{\max }-n_{\min }$, where $n_{\max }\left(n_{\min }\right)$ can be found from the conditions $\Gamma_{3}^{(-)}(n) \geqslant 0 \quad\left(\Gamma_{1}^{(+)}(n) \geqslant 0\right)$. The value $e n_{\max }$, (e $\left.n_{\min }\right)$ gives the maximum (minimum) charge that can be in the island for a given voltages $U_{1}, U_{2}$, and $V_{g}$.

We can see from Eq. (47) that there are four elementary processes of charge transfer in the system at low temperatures, each of them is being associated with the prefactor $e^{ \pm i \chi_{k}}$. The presence of the exponents $e^{i \chi_{3}}$ and $e^{-i \chi_{2}}$ corresponds to the charge transfer from the third terminal into the island and from the island into the second terminal, respectively. Hence, the random current through the third (second) junctions always has the positive (negative) sign. Two factors $e^{ \pm \chi_{1}}$ stems from the charge transfer through the first junction in the direction either from the island into the first contact or vice versa. Therefore, the random current $I_{1}$ is able to fluctuate in both directions.

Consider first the shot-noise correlations in the system. For that it is useful to introduce the $(3 \times 3)$ matrix $F$ with elements $F_{k m}=S_{k m} / e I_{\Sigma}$, where the current correlations $S_{k m}$ are given by Eq. (44) and $I_{\Sigma}=\sum_{i=1}^{3}\left|I_{i}\right|$. The matrix $F$ is a generalization of the Fano factor for the multiterminal system. It is symmetric and obeys the relation $\sum_{i=1}^{3} F_{i k}=0$. It follows from the general law of the current conservation in the system. For the considered three-terminal dot we have found that the cross-correlations $F_{k m}(k \neq m)$ are always negative for any set of parameters.

In Fig. 11 we give the illustrative example of the voltage dependence of the shot-noise correlations $F_{k m}$ for the certain choice of parameters. As in the two-terminal case the Coulomb blockade features are strongly pronounced only for the asymmetric setup. The results in Fig. 10 corresponds to $R_{1}$ $=R_{3}=R_{2} / 10, \widetilde{C}_{1}=\widetilde{C}_{2}=\widetilde{C}_{3}$, and $U_{2} / U_{1}=4$. The latter ratio of voltages has been chosen on the basis of arguments that for a given value of resistances $R_{k}$ it would split the average current $I_{3}$ into two equal currents $\left|I_{1}\right|=\left|I_{2}\right|=I_{3} / 2$ provided 


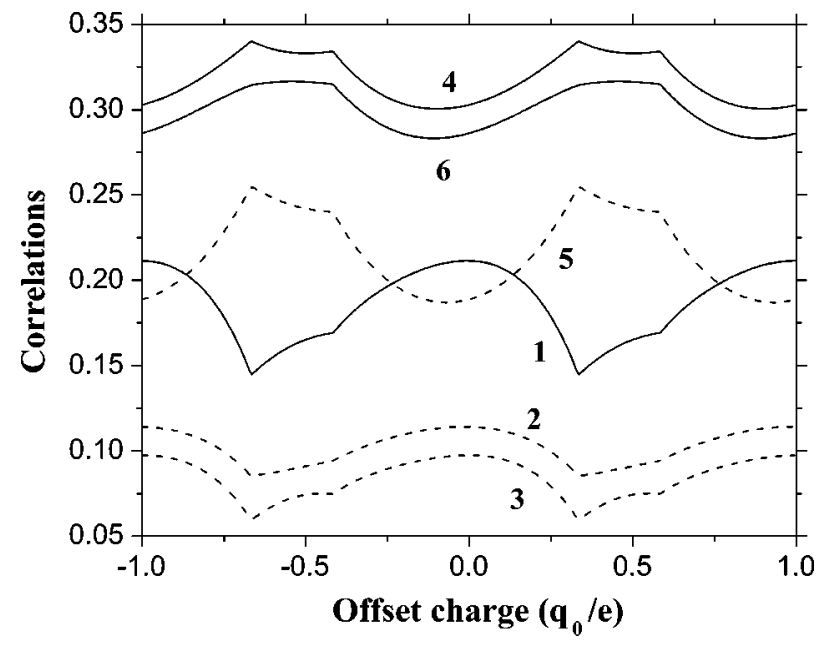

FIG. 12. The matrix $F$ of auto- and cross-shot-noise correlation versus the offset charge for the three-terminal quantum dot setup. Parameters are the same as on Fig. 11. The voltage $U_{1}=U_{2} / 4$ $=1.25 e / C_{\Sigma}$. (1) $F_{11}$, (2) $\left|F_{12}\right|$, (3) $\left|F_{13}\right|$, (4) $F_{22}$, (5) $\left|F_{23}\right|$, (6) $F_{33}$.

one could apply the usual linear Kirchgoff rules to this circuit. In Fig. 12 the dependence of the shot noise correlations on the offset charge is shown for the same set of parameters and the value of $U_{1}=1.25 e / C_{\Sigma}$. The special points of both these dependences occur when either $n_{\min }, n_{\max }$ or the integer part of $q$ are changed by \pm 1 . As the result we observe multiperiodic Coulomb blockade oscillations in the offset charge dependences in contrast to the single periodic oscillations in the two-terminal case.

We now proceed with the consideration of the FCS. As before, the action $S\left(\left\{\chi_{i}\right\}\right)$ has been calculated with the use of Eq. (27). Afterwards probability (18) has been estimated by

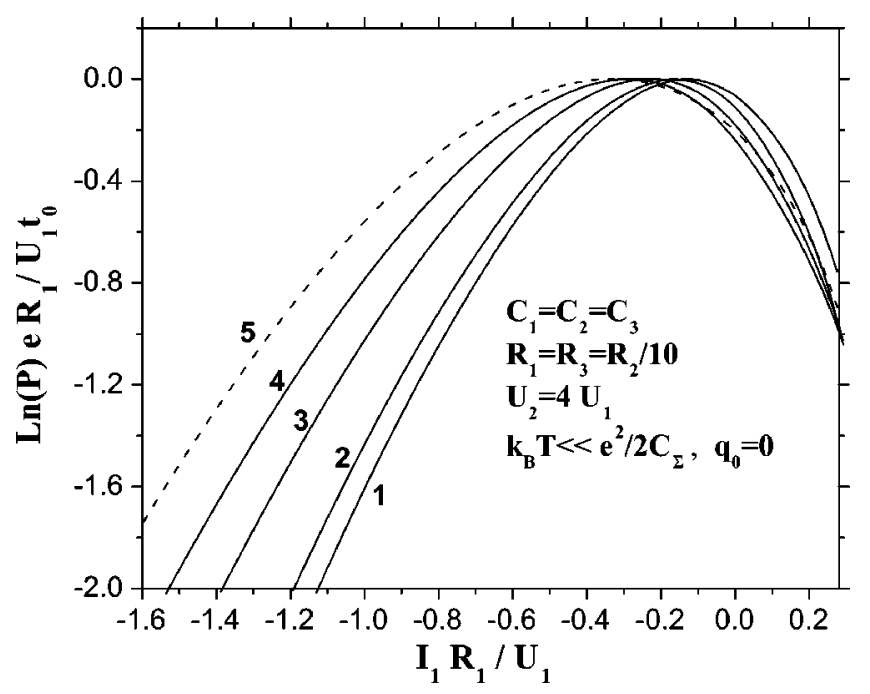

FIG. 13. The logarithm of current distribution $\ln P\left(I_{1}, I_{2}\right)$ in the three-terminal quantum dot as a function of current $I_{1}$, under condition $I_{2}=\left\langle I_{2}\right\rangle$. Parameters are shown on the plot. (1) $-U_{1}$ $=1.25 \mathrm{e} / C_{\Sigma}$, (2) $U_{1}=2.0 \mathrm{e} / C_{\Sigma}$, (3) $U_{1}=4.0 \mathrm{e} / C_{\Sigma}$, (4) $U_{1}$ $=10.0 \mathrm{e} / C_{\Sigma}$, and curve (5) corresponds to the noninteracting regime.

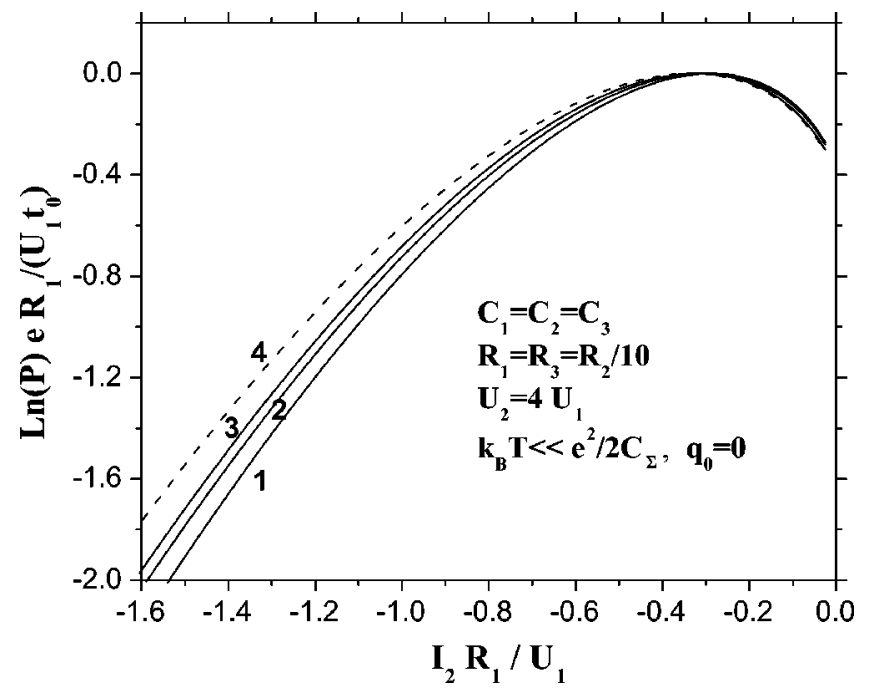

FIG. 14. The logarithm of current distribution $\ln P\left(I_{1}, I_{2}\right)$ in the three-terminal quantum dot as a function of current $I_{2}$, under condition $I_{1}=\left\langle I_{1}\right\rangle$. Parameters are the same as in Fig. 13. (1) $U_{1}$ $=1.25 e / C_{\Sigma}$, (2) $U_{1}=2.0 e / C_{\Sigma}$, (3) $U_{1}=4.0 e / C_{\Sigma}$, and curve (4) corresponds to the noninteracting regime.

means of the steepest descent method. The difference with the two-terminal geometry is that three currents $I_{k}$ and three counting fields $\chi_{k}$ are now involved. Due to the current conservation $\Sigma_{k} I_{k}=0$ for any plausible fluctuation, only two currents are independent. Thus the action $S\left(\left\{\chi_{i}\right\}\right)$ depends on the differences $\chi_{i j}=\chi_{i}-\chi_{j}$ only. In what follows we have chosen $I_{1}$ and $I_{2}$ as the independent variables to plot the logarithm of probability $\ln P\left(I_{1}, I_{2}\right)$. With the exponential accuracy it is given by $\ln P\left(I_{1}, I_{2}\right) \sim e^{-\Omega\left(\chi^{*}\right)}$. Here $\chi^{*}$ is a saddle point of the function $\Omega(\chi)=S(\chi)+i \chi_{1} I_{1} t_{0} / e+i \chi_{2} I_{2} t_{0} / e$. The results for $\ln P\left(I_{1}, I_{2}\right)$ are shown in Figs. 13-15. From the contour map on Fig. 15 we see that $P\left(I_{1}, I_{2}\right)$ is nonzero in the quarter $I_{1}<0, I_{2}<0$ of a current plain $\left(I_{1}, I_{2}\right)$ and in the region $I_{1} \leqslant\left|I_{2}\right|$ provided $I_{1}>0, I_{2}<0$. This range of plausible current fluctuations stems from the $\chi$-dependence of rates Eq. (47). Any current fluctuation automatically satisfies the constrain $\Sigma_{k} I_{k}=0$ and conditions $I_{2}<0$ and $I_{3}>0$.

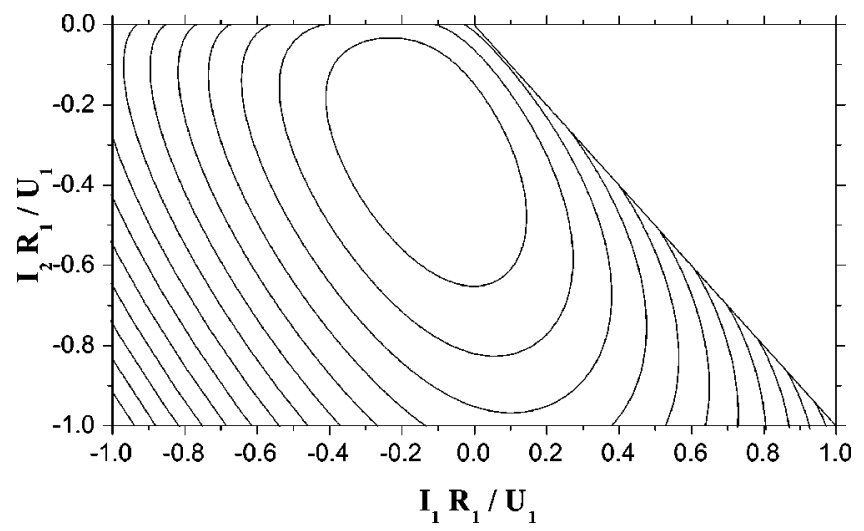

FIG. 15. The contour maps of the current distribution $\log \left[P\left(I_{1}, I_{2}\right)\right]$ in the three-terminal Coulomb blockade island. Parameters are the same as in Fig. 13. $U_{1}=U_{2} / 4.0=1.25 \mathrm{e} / C_{\Sigma}$. 
Before discussing the above results it is worth to setup the reference point for such discussion. This reference will be our previous results ${ }^{11}$ for the FCS in the three-terminal chaotic quantum dot when its contact are tunnel junctions with resistances $R_{k}^{-1} \gg e^{2} / \pi \hbar$. In this limit the effects of interaction are negligible and electrons are scattered independently at different energies. Provided $U_{2}>U_{1}$, the generating function $S\left(\left\{\chi_{i}\right\}\right)$ in the given case is a sum of the two independent processes

$$
S\left(\chi_{1}, \chi_{2}, \chi_{3}\right)=S_{1}\left(\chi_{1}, \chi_{2}, \chi_{3}\right)+S_{2}\left(\chi_{1}, \chi_{2}, \chi_{3}\right) .
$$

Here

$$
\begin{gathered}
S_{1}\left(\chi_{1}, \chi_{2}, \chi_{3}\right)=\frac{U_{1} t_{0}}{2 e}\left\{G_{1}+G_{2}+G_{3}-\sqrt{\left(G_{1}+G_{2}-G_{3}\right)^{2}+4 G_{3} e^{i \chi_{3}}\left(G_{1} e^{\left.-i \chi_{1}+G_{2} e^{-i \chi_{2}}\right)}\right\},}\right. \\
S_{2}\left(\chi_{1}, \chi_{2}, \chi_{3}\right)=\frac{\left(U_{2}-U_{1}\right) t_{0}}{2 e}\left\{G_{1}+G_{2}+G_{3}-\sqrt{\left(G_{1}+G_{3}-G_{2}\right)^{2}+4 G_{2} e^{-i \chi_{2}}\left(G_{1} e^{i \chi_{1}}+G_{3} e^{i \chi_{3}}\right)}\right\},
\end{gathered}
$$

and $G_{k}=R_{k}^{-1}$ are the conductances of the junctions.

The logarithm of probability $\ln P_{0}\left(I_{1}, I_{2}\right)$, evaluated with the use of statistics (50), is shown by the dashed line in addition to the previous curves in Figs. 13 and 14. Its contour map for the same values of parameters is also separately presented in Fig. 16. The maximum of $\ln P_{0}\left(I_{1}, I_{2}\right)$, as expected, occur at $\bar{I}_{1}=\bar{I}_{2}=U_{1} / 3 R_{1}$.

We can derive the following conclusions on comparing the FCS in the Coulomb blockade and noninteracting limits. In spite of the different regimes, we see that the qualitative dependence of probabilities versus the currents is similar for both statistics. The probability distribution in both cases has a single maximum, corresponding to the average values of currents. The tails of distribution are essentially nonGaussian both in the weak and strong interacting limit. The statistics approaches to the Gaussian-type one in the strong Coulomb blockade limit only, when the applied voltage to the system is only few above the Coulomb blockade threshold. [See curves (1) and (2) in Fig. 13.] At higher applied voltages the probability distribution has a tendency to approach to the current distribution of the noninteracting system. However, they never become identical, even in the limit $U_{1,2} \gg e / C_{\Sigma}$. [Curves (4) and (5).] The same is true for the shot-noise correlations. Generally, we conclude, that the

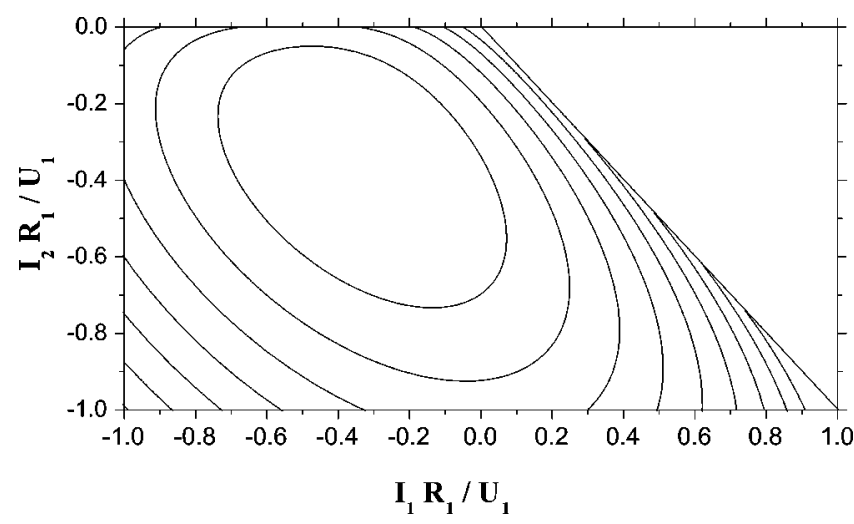

FIG. 16. The contour maps of the current distribution $\log \left[P\left(I_{1}, I_{2}\right)\right]$ in the three-terminal chaotic quantum dot with tunnel contacts. $U_{1}=U_{2} / 4.0=1.25 \mathrm{e} / C_{\Sigma}$.
Coulomb interaction always suppresses the relative probabilities of big current fluctuations. This behavior stems from the fact, that any big current fluctuation in Coulomb blockade island is related with the large accumulation (or depletion) of the charge on the island. The latter process results in the excess of electrostatic energy. Therefore, the relative probability of such fluctuation is decreased, as compared to the probability of the similar current fluctuation in the noninteracting regime.

\section{CONCLUSIONS}

To conclude, in the present paper we have developed the constructive scheme to evaluate the FCS of charge transfer in the Coulomb blockade systems. This scheme is rather general and universal and is applicable to any strongly interacting system, provided the latter can be described classically in the framework of the master equation approach. The method proposed consists in the transformation of the initial linear operator $\hat{L}$ of the master equation into the auxiliary $\chi$-dependent linear operator $L_{\chi}$. Each nondiagonal term of this new operator, associated with the particular transition in the system, is modified by the exponential prefactor $e^{ \pm i \chi_{k}}$ in order to take into account the electron jump through the junction $k$ during the tunneling event. The generating function of the charge transfer through the whole system is then proportional to the minimal eigenvalue of the operator $L_{\chi}$.

We have applied this scheme to study the FCS in two different Coulomb blockade systems. For a generic case of a single resonant-level model we have established the equivalence of the developed method with the scattering approach to the FCS, when the particles in the system are noninteracting.

Afterwards we have considered the FCS and the shotnoise correlations in the two- and three-terminal Coulomb blockade islands. The consideration was limited to the temperature regime $k_{B} T \gg \Delta E$, with $\Delta E$ being the mean level spacing in the dot. In this regime we made use of the "orthodox theory" of Coulomb blockade phenomenon. For the case of two-terminal Coulomb blockade island we have reestablished all the known results for the shot-noise in this system. For the Coulomb blockade island with three leads 
attached we have shown that the auto- and cross-shot-noise correlations exhibit the characteristic Coulomb blockade oscillations as the functions of the applied voltages and the offset charge.

We have considered the question of the FCS in the above two types of dots as well. In the general situation we evaluated the probability distribution numerically. However, we have managed to find FCS analytically in case of twoterminal Coulomb blockade island at some special values of parameters. In these exceptional cases the FCS resembles the statistics of the charge transfer through the single resonant level. Then we compared the statistics in the Coulomb blockade island with our previous results concerning an open chaotic quantum dot with two and three terminals. We found that the Coulomb interaction suppresses the relative probability of the big current fluctuations.

\section{ACKNOWLEDGMENTS}

We are grateful to M. Ueda for bringing our attention to Refs. 28-30. This work was a part of the research program of the "Stichting voor Fundamenteel Onderzoek der Materie" (FOM), and we acknowledge the financial support from the "Nederlandse Organisatie voor Wetenschappelijk Onderzoek" (NWO).

\section{APPENDIX A}

In this Appendix we show that the construction of the probability measure on the basis of Markov chains $\zeta_{s}$, which was used to derive the main result of Sec. III, leads to the usual description of the system dynamics in terms of master equation. This correspondence is achieved in the standard way of probability theory by introducing the stochastic process $\check{n}(t)$ corresponding to the island charge at a given time $t$

$$
n\left(t, \zeta_{s}\right)=n_{s}+\sum_{i=1}^{s} \sigma_{i} \theta\left(t-\tau_{i}\right)
$$

Similarly one can consider the random number of electrons $\check{n}^{(k)}(t)$ transferred through the junction $k$ after $t \geqslant-T / 2$

$$
n^{(k)}\left(t, \zeta_{s}\right)=\sum_{i=1}^{s} \sigma_{i} \theta\left(t-\tau_{i}\right) \delta\left(k-k_{i}\right)
$$

The random variables $\check{n}(t)$ and $\check{n}^{(k)}(t)$ are subjected to the relations

$$
\begin{gathered}
n\left(t, \zeta_{s}\right)=n_{s}+\sum_{k=1}^{N} n^{(k)}\left(t, \zeta_{s}\right), \\
I^{(k)}\left(t, \zeta_{s}\right)=e \frac{\partial}{\partial t} n^{(k)}\left(t, \zeta_{s}\right) .
\end{gathered}
$$

After that we can introduce the probability distribution $P(n, t)$ and the joint probability distribution $P\left(n_{1}, t_{1} ; n_{2}, t_{2}\right)$ $\left(t_{1} \geqslant t_{2}\right)$ of the process $\check{n}(t)$

$$
P(n, t)=\int_{\Omega} \delta(n-n(t, \zeta)) d \mu(\zeta)
$$

$P\left(n_{1}, t_{1} ; n_{2}, t_{2}\right)=\int_{\Omega} \delta\left[n_{1}-n\left(t_{1}, \zeta\right)\right] \delta\left[n_{2}-n\left(t_{2}, \zeta\right)\right] d \mu(\zeta)$.

Their ratio $P\left(n_{1}, t_{1} \mid n_{2}, t_{2}\right)=P\left(n_{1}, t_{1} ; n_{2}, t_{2}\right) / P\left(n_{2}, t_{2}\right)$ gives the conditional probability to find the system at state $n_{1}$ at time $t_{1}$, given that at time $t_{2}$ it was at state $n_{2}$. The integrals, Eq. (A4), can be efficiently evaluated along with the same reasoning as we have used to prove the normalization condition. As the result one ends up with

$$
P\left(n_{1}, t_{1} \mid n_{2}, t_{2}\right)=\left\langle n_{1}\left|\hat{U}\left(t_{1}, t_{2}\right)\right| n_{2}\right\rangle
$$

The latter expression is the usual way to describe the system in terms of master equation. The conditional probability $P\left(n_{1}, t_{1} \mid n_{2}, t_{2}\right)$ regarded as a function of $n_{1}$ and $t_{1}$ obeys this equation with the initial condition $P\left(n_{1}, t_{1}\right)=\delta_{n_{1}, n_{2}}$ at $t_{1}=t_{2}$.

\section{APPENDIX B}

This appendix contains the derivation of the action $S(\chi)$ at low temperatures at some special points $V_{k}$ in the Coulomb blockade staircase in the two-terminal island. We introduce the notation $\Gamma_{\chi}^{( \pm)}(n)=\Gamma_{n \pm \leftarrow n}^{\chi}$, that enables to write down Eq. (43) in the form

$$
\left(\Lambda-\gamma_{n}\right) p_{n}+\Gamma_{\chi}^{(-)}(n+1) p_{n+1}+\Gamma_{\chi}^{(+)}(n-1) p_{n-1}=0 .
$$

If all $\chi_{k}=0$ then the stationary solution of this equation, corresponding to $\Lambda=0$, satisfies the detailed balance condition $p_{n+1} \Gamma_{0}^{(-)}(n+1)=p_{n} \Gamma_{0}^{(+)}(n)$. In general situation, when $\chi_{k} \neq 0$, one may try to resolve Eq. (B1) making use of the substitution

$$
\frac{p_{n+1}}{p_{n}}=\frac{\Gamma_{\chi}^{(+)}(n)}{y \Gamma_{\chi}^{(-)}(n+1)}, \quad \frac{p_{n-1}}{p_{n}}=\frac{y \Gamma_{\chi}^{(-)}(n)}{\Gamma_{\chi}^{(+)}(n-1)}
$$

with unknown constant $y$ to be found. This reduces the difference Eq. (B1) to the relation

$$
\left(\Lambda-\gamma_{n}\right)+\Gamma_{\chi}^{(-)}(n) y+\Gamma_{\chi}^{(+)}(n) y^{-1}=0 .
$$

Here $\gamma_{n}=\Gamma_{0}^{(-)}(n)+\Gamma_{0}^{(+)}(n)$, and $\Gamma_{\chi}^{( \pm)}(n)$ are linear functions in $n$, given by Eq. (45). Then one might find the two unknown $y$ and $\Lambda_{\chi}$ on comparing the constant and linear in $n$ terms in relation (B3). It yields

$$
\begin{gathered}
y=\left(R_{1}^{-1}-R_{2}^{-1}+\sqrt{\mathcal{D}(\chi)}\right) / 2 R_{1}^{-1} e^{-i \chi_{1}}, \\
\Lambda(\chi)=\frac{1}{2 e}\left(V-e / C_{\Sigma}\right)\left[R_{1}^{-1}+R_{2}^{-1}-\sqrt{\mathcal{D}(\chi)}\right] \\
\mathcal{D}(\chi)=\left(R_{1}^{-1}-R_{2}^{-1}\right)^{2}+4\left(R_{1} R_{2}\right)^{-1} e^{i\left(\chi_{2}-\chi_{1}\right)} .
\end{gathered}
$$

It looks like we have found in such a way the required solution. However, it is not valid at all possible values of param- 
eters. The matter is that the expressions [Eq. (45)] are not correct at points $n=n_{\min }$ and $n=n_{\text {max }}$ in the most general case. One has to satisfy the boundary condition $\Gamma_{\chi}^{(-)}\left(n_{\max }\right)$ $=\Gamma_{\chi}^{(+)}\left(n_{\min }\right)=0$. This violates the analytical $n$ dependence of Eqs. (B1) and (B3) at the boundaries. The only exceptional situation, when expressions (B2) and (B4) solve the Eq.
(B1), corresponds to the case $n_{1}=n_{\min }, n_{2}=n_{\text {max }}$, with $n_{1(2)}$ being the zeros of functions $\Gamma_{\chi}^{( \pm)}(n)$. In this case the substitution Eq. (B2) gives $p\left(n_{\max }+1\right)=p\left(n_{\min }-1\right)=0$ and hence the actual values of $\Gamma_{\chi}^{(-)}\left(n_{\max }+1\right)$ and $\Gamma_{\chi}^{(+)}\left(n_{\min }-1\right)$ in Eq. (B1) play no role. Then we arrive to the action $S(\chi)$ $=t_{0} \Lambda(\chi)$ in the form which was claimed in Sec. $\mathrm{V}(\mathrm{b})$.
${ }^{1}$ Ya.M. Blanter and M. Büttiker, Phys. Rep. 336, 1 (2000).

${ }^{2}$ L.S. Levitov and G.B. Lesovik, Pis'ma Zh. Éksp. Teor. Fiz. 58, 225 (1993) [JETP Lett. 58, 230 (1993)]; L.S. Levitov, H.-W. Lee, and G.B. Lesovik, J. Math. Phys. 37, 4845 (1996).

${ }^{3}$ H. Lee, L.S. Levitov, and A.Yu. Yakovets, Phys. Rev. B 51, 4079 (1995).

${ }^{4}$ Ya.M. Blanter, H. Schomerus, and C.W.J. Beenakker, Physica E (Amsterdam) 11, 1 (2001).

${ }^{5}$ A. Andreev and A. Kamenev, Phys. Rev. Lett. 85, 1294 (2000).

${ }^{6}$ L.S. Levitov, arXiv:cond-mat/0103617 (unpublished).

${ }^{7}$ Y. Makhlin and A.D. Mirlin, Phys. Rev. Lett. 87, 276803 (2001).

${ }^{8}$ Yu.V. Nazarov, Special issue of Comments Ann. Phys. (Leipzig) 8, SI-193 (1999).

${ }^{9}$ Yu. V. Nazarov, in Quantum Dynamics of Submicron Structures, edited by H. Cerdeira, B. Kramer, and G. Schoen (Kluwer Academic, Dordrecht, 1995), p. 687.

${ }^{10}$ W. Belzig and Yu.V. Nazarov, Phys. Rev. Lett. 87, 067006 (2001); W. Belzig and Yu.V. Nazarov, ibid. 87, 197006 (2001).

${ }^{11}$ Yu.V. Nazarov and D.A. Bagrets, Phys. Rev. Lett. 88, 196801 (2002).

${ }^{12}$ J. Borlin, W. Belzig, and C. Bruder, Phys. Rev. Lett. 88, 197001 (2002).

${ }^{13}$ A.V. Andreev and E.G. Mishchenko, Phys. Rev. B 64, 233316 (2001).

${ }^{14}$ A.N. Korotkov, Phys. Rev. B 49, 10381 (1994).

${ }^{15}$ S. Hershfield, J.H. Davis, P. Hyldgaard, C.J. Stanton, and J. Wilkins, Phys. Rev. B 47, 1967 (1993).
${ }^{16}$ U. Hanke, Yu.M. Galperin, K.A. Chao, and N. Zhou, Phys. Rev. B 48, 17209 (1993).

${ }^{17}$ U. Hanke, Yu.M. Galperin, and K.A. Chao, Phys. Rev. B 50, 1595 (1994).

${ }^{18}$ B.R. Bulka, J. Martinek, G. Michalek, and J. Barnaś, Phys. Rev. B 60, 12246 (1999).

${ }^{19}$ H. Birk, M.J.M. de Jong, and C. Schönenberger, Phys. Rev. Lett. 75, 1610 (1995).

${ }^{20}$ A.I. Larkin and K.A. Matveev, Zh. Éksp. Teor. Fiz. 93, 1030 (1987) [Sov. Phys. JETP 66, 580 (1987)].

${ }^{21}$ L.I. Glazman and K.A. Matveev, Pis'ma Zh. Éksp. Teor. Fiz. 48, 403 (1988) [JETP Lett. 48, 445 (1988)].

${ }^{22}$ V.V. Kuznetsov, A.K. Savchenko, D.R. Mace, E.H. Linfield, and D.A. Ritchie, Phys. Rev. B 56, R15 533 (1997).

${ }^{23}$ C.W.J. Beenakker, Phys. Rev. B 44, 1646 (1991).

${ }^{24}$ G.-L. Ingold and Yu. V. Nazarov, in Single Charge Tunneling, Vol. 294 of NATO Series B: Physics, edited by H. Grabert and M. H. Devoret (Plenum Press, New York, 1992).

${ }^{25}$ N. G. van Kampen, Stochastic Processes in Physics and Chemistry, Rev. and enl. ed. (Elsevier Scinece Publishes B. V., NorthHolland, 1992).

${ }^{26}$ Yu.V. Nazarov and J.J.R. Struben, Phys. Rev. B 53, 15466 (1996).

${ }^{27}$ L. Mandel and E. Wolf, Optical Coherence and Quantum Optics (Cambridge University Press, Cambridge, 1995).

${ }^{28}$ M. Ueda, Phys. Rev. A 38, 2937 (1988).

${ }^{29}$ M. Ueda, Phys. Rev. A 40, 1096 (1989).

${ }^{30}$ A. Shimizu and M. Ueda, Phys. Rev. Lett. 69, 1403 (1992). 